\title{
$\kappa$-Minkowski spacetime and the star product realizations
}

\author{
S. Meljanac ${ }^{1}$, A. Samsarov ${ }^{2}$ and M. Stojić ${ }^{3}$ \\ Rudjer Bošković Institute, Bijenička c.54, HR-10002 Zagreb, Croatia \\ Kumar S. Gupta 4 \\ Theory Division, Saha Institute of Nuclear Physics, 1/AF, Bidhannagar, Calcutta 700064, India
}

\begin{abstract}
We investigate a Lie algebra-type $\kappa$-deformed Minkowski spacetime with undeformed Lorentz algebra and mutually commutative vector-like Dirac derivatives. There are infinitely many realizations of $\kappa$-Minkowski space. The coproduct and the star product corresponding to each of them are found. An explicit connection between realizations and orderings is established and the relation between the coproduct and the star product, provided through an exponential map, is proved. Utilizing the properties of the natural realization, we construct a scalar field theory on $\kappa$-deformed Minkowski space and show that it is equivalent to the scalar, nonlocal, relativistically invariant field theory on the ordinary Minkowski space. This result is universal and does not depend on the realizations, i.e. orderings used.
\end{abstract}

\section{INTRODUCTION}

One of the problems whose solution is still being sought involves how gravity and quantum field theory can be reconciled. The efforts to bring these two theories closer have resulted in many new developments in theoretical physics and mathematics. In this regard, noncommutative geometry appears as a promising framework for unifying gravity and quantum field theory. In order to accomodate for quantum aspects of spacetime, spacetime noncommutativity has been studied extensively [1], [2], [3], [4], [5], [6] and two main directions in the investigation have focused on the canonical noncommutative spacetime and $\kappa$-Minkowski noncommutative spacetime [7]-[32], respectively.

To describe the short-distance (Planck scale) structure of spacetime, there appeared a possible necessity for modification of the existing symmetries. Specifically, it might require some deformation of Poincare symmetry and some arguments would suggest that the symmetries of the $\kappa$-deformed Minkowski space should be described in terms of a Hopf algebra [8], 10]. For these reasons, it is of importance to classify noncommutative (NC) spaces and to investigate their properties. In order to make a step forward in this direction, we analyze a $\kappa$-deformed Minkowski space which is a simple example of a Lie algebra type $\mathrm{NC}$ space [33].

Recently, a new motivation for studying $\kappa$-deformed spacetime has appeared within the programme of the so-called doubly special relativity (DSR) [16],[17], which is a special relativity theory extended to include a second invariant parameter $\kappa$, a parameter of mass dimension, besides the velocity of light. Also, $\kappa$-Minkowski space serves as a playground for constructing a field theory on it and then discussing its physical properties. The attempts of this type have been undertaken by many authors [7]- 32 .

In $\kappa$-deformed Minkowski space, the noncommutativity of coordinates depends on a deformation parameter $a=\frac{1}{\kappa}$. Generally, this parameter can be considered as an arbitrary vector $a$ in Minkowski space with all components different from zero. In Refs. 24] and [25], the most general deformation of the Euclidean space with the vector $a$ in an arbitrary direction has been discussed. In the present paper we restrict to the case where only the time component of the vector $a$ is different from zero. The dimensional parameter $a=\frac{1}{\kappa}$ has a very small value which yields the undeformed Minkowski space in the limit $a \rightarrow 0$. The NC coordinates and the generators of rotations form an extended Lie algebra.

\footnotetext{
1 e-mail: meljanac@irb.hr

2 e-mail: asamsarov@irb.hr

3 e-mail: marko.stojic@zg.htnet.hr

4 e-mail: kumars.gupta@saha.ac.in
} 
The subalgebra which includes the rotation generators is the ordinary undeformed Lorentz algebra $S O_{a}(1, n-1)$. We assume that Dirac derivatives mutually commute and transform as a vector representation of the Lorentz algebra. An infinite number of realizations [24], 25] of $\kappa$-deformed Minkowski space is found in terms of commutative coordinates and derivatives. These realizations are parametrized by arbitrary invertible functions, with suitably imposed conditions leading to the undeformed Minkowski space in the limit $a \rightarrow 0$. Each such realization induces an ordering prescription, a coproduct and a star product associated to it. For a special choice of the parameter functions, some particularly simple realizations are obtained whose corresponding star products are known in the literature 24]. The choice of the particular realization in a way corresponds to a particular choice of gauge, so although $\kappa$-Minkowski space may be realized in many different ways, physical results do not depend on concrete realization, i.e. ordering, as long as all commutators between NC coordinates and the Dirac derivatives are fixed. In the analyzis of $\kappa$-Minkowski spaces we use the methods developed for deformed single and multimode oscillators in the Fock space representations [34]-[40]. In particular, we use the methods for constructing deformed creation and annihilation operators in terms of ordinary bosonic multimode oscillators. Also, we employ the construction of transition number operators and, in general, of generators proposed in Refs. [35], 36], 38].

The crucial point emphasized in this paper is that NC spaces, particularly the $\kappa$-deformed Minkowski space considered here, can be realized in many ways in terms of commutative variables and all these realizations are on equal footing with physical results not depending on the particular realization taken. For fixed deformed Heisenberg algebra, there is a one-to-one correspondence between the realization of $\mathrm{NC}$ space and the ordering prescription and for each such realization there exists a coproduct and a particular star product corresponding to it. In a recent paper [30] it was shown that a free scalar field theory on $\kappa$-deformed Minkowski space can be expressed as a nonlocal, relativistically invariant field theory on the ordinary Minkowski space. The authors of the Ref. [30] defined a special star product by using, from the very beginning, a particular "right" ordering prescription. In the present paper we also construct a free scalar field theory on $\kappa$-deformed Minkowski space in terms of a particularly chosen star product, namely the star product corresponding to the natural realization, where the Dirac derivative is identified with the ordinary derivative. We show that the corresponding free scalar field theory on Minkowski space is particularly simple, nonlocal, relativistically invariant and does not depend on the realization/ordering taken, i.e. does not depend on the particular realization of 4 -momenta and coordinates defining the related $\mathrm{NC}$ space.

This paper is arranged as follows. In section 2.1 we review the results of Ref. 24] which are important for the investigations carried out in this paper, including the notions of $\kappa$-deformed Euclidean space, its realizations in terms of commutative coordinates, and the realizations of the undeformed rotation algebra $S O_{a}(n)$ compatible with $\kappa$ deformation. All these realizations are introduced in such a way as to retain the structure of the extended Lie algebra. They describe the Lie algebra type deformation of space where the rotation algebra is undeformed, while the corresponding coalgebra is deformed. The Dirac derivative and the invariant Klein Gordon operator, together with their realizations, are also introduced in this section. In section 2.3 we exhibit the one-to-one correspondence between the ordering precription and the type of mapping between the NC $\kappa$-deformed space and the ordinary undeformed space. Here we also give the general $\mathrm{BCH}$ formula for the Lie algebra corresponding to the $\kappa$ type of deformation of space. In section 2.4 the notions of the T- operator and a star product are introduced. Knowing the star product in a given realization, the $\mathrm{T}$ - operator can be used to make a transition to another, equivalent star product. This new star product corresponds to a realization which is related to first one through a similarity transformation by the Toperator. In section 3, a derivation of the star product formula corresponding to the $\varphi$ realization, introduced in section 2.1, is presented. The final result is identified with an important relation between the coproduct and the star product in terms of an exponential map for a given realization. This result can be extended to hold for a general Lie algebra. In section 4 all results obtained are continued to the Minkowski space with suitable modifications required to accomodate for a change in the signature. Here we introduce a particularly interesting realization, the so-called natural realization, discuss its properties and derive the star product corresponding to it. We then use this result to construct the free scalar field theory on the $\kappa$-deformed Minkowski space and to show that it reduces to free, nonlocal scalar field theory on the ordinary Minkowski spacetime, no matter which realization of the deformed space is used. In constructing the field theory on $\kappa$-deformed Minkowski space, the emphasis is on the star product corresponding 
to the natural realization because it is the only one with this preferable property. After a short summary, there are two Appendices which refer mainly to the derivation of the star product, taking place in section 3 .

\section{ALGEBRA OF $\kappa$ - DEFORMED EUCLIDEAN SPACE}

\subsection{The mapping between commutative and noncommutative coordinates}

We consider a noncommutative (NC) space [24] with coordinates $\hat{x}_{1}, \hat{x}_{2}, \ldots, \hat{x}_{n}$, satisfying the following Lie algebra type relations:

$$
\left[\hat{x}_{\mu}, \hat{x}_{\nu}\right]=i C_{\mu \nu \lambda} \hat{x}_{\lambda}=i\left(a_{\mu} \hat{x}_{\nu}-a_{\nu} \hat{x}_{\mu}\right)
$$

where $a_{1}, a_{2}, \ldots, a_{n}$ are constant real parameters describing a deformation of Euclidean space. The structure constants are

$$
C_{\mu \nu \lambda}=a_{\mu} \delta_{\nu \lambda}-a_{\nu} \delta_{\mu \lambda}
$$

We choose $a_{1}=a_{2}=\ldots=a_{n-1}=0, a_{n}=a$ and use Latin indices for the subspace $(1,2, \ldots, n-1)$ and Greek indices for the whole space $(1,2, \ldots, n)$. Then the algebra of the NC coordinates becomes

$$
\left[\hat{x}_{i}, \hat{x}_{j}\right]=0, \quad\left[\hat{x}_{n}, \hat{x}_{i}\right]=i a \hat{x}_{i}, \quad i, j=1,2, \ldots, n-1
$$

There exist realizations of the NC coordinates $\hat{x}_{\mu}$ in terms of ordinary commutative coordinates $x_{1}, x_{2}, \ldots, x_{n}$ and their derivatives $\partial_{1}, \partial_{2}, \ldots, \partial_{n}$, where $\partial_{\mu}=\frac{\partial}{\partial x_{\mu}}$. A realization of the NC coordinates $\hat{x}_{\mu}$ satisfying the algebra (3) is

$$
\begin{gathered}
\hat{x}_{i}=x_{i} \varphi(A), \\
\hat{x}_{n}=x_{n} \psi(A)+i a x_{k} \partial_{k} \gamma(A),
\end{gathered}
$$

where $A=i a \partial_{n}$ and the summation over repeated indices is understood. It is assumed that $\varphi$ and $\psi$ are positive functions obeying the conditions

$$
\varphi(0)=1, \quad \psi(0)=1
$$

The function $\gamma$ satisfy

$$
\frac{\varphi^{\prime}}{\varphi} \psi=\gamma-1
$$

where $\varphi^{\prime}=\frac{d \varphi}{d A}$. It is understood that the quantity $\gamma(0)=\varphi^{\prime}(0)+1$ is finite. In the following discussion we take the vacuum state to be represented by 1 , i.e. $|0\rangle \equiv 1$, so that it is annihilated by all derivatives $\partial_{\mu}$.

Next we introduce the generators $M_{\mu \nu}, M_{\mu \nu}=-M_{\nu \mu}$ satisfying the ordinary undeformed $S O_{a}(n)$ algebra

$$
\left[M_{\mu \nu}, M_{\lambda \rho}\right]=\delta_{\nu \lambda} M_{\mu \rho}-\delta_{\mu \lambda} M_{\nu \rho}-\delta_{\nu \rho} M_{\mu \lambda}+\delta_{\mu \rho} M_{\nu \lambda}
$$

or in a more practical form

$$
\begin{gathered}
{\left[M_{i j}, M_{j n}\right]=M_{i n},} \\
{\left[M_{i n}, M_{j n}\right]=-M_{i j} .}
\end{gathered}
$$

If we want to extend the algebra to include the action of the generalized rotation generators $M_{\mu \nu}$ on NC coordinates in such a way as to keep the Lie algebra structure, then we are brought to the unique commutation relations [20], 24]

$$
\left[M_{i n}, \hat{x}_{n}\right]=\hat{x}_{i}+i a M_{i n}
$$




$$
\left[M_{i n}, \hat{x}_{j}\right]=-\delta_{i j} \hat{x}_{n}+i a M_{i j}
$$

that, together with (3) and (7), satisfy the Jacobi identities

$$
\begin{gathered}
{\left[M_{\alpha \beta},\left[\hat{x}_{\mu}, \hat{x}_{\nu}\right]\right]+\left[\hat{x}_{\mu},\left[\hat{x}_{\nu}, M_{\alpha \beta}\right]\right]+\left[\hat{x}_{\nu},\left[M_{\alpha \beta}, \hat{x}_{\mu}\right]\right]=0,} \\
{\left[M_{\alpha \beta},\left[M_{\gamma \delta}, \hat{x}_{\mu}\right]\right]+\left[M_{\gamma \delta},\left[\hat{x}_{\mu}, M_{\alpha \beta}\right]\right]+\left[\hat{x}_{\mu},\left[M_{\alpha \beta}, M_{\gamma \delta}\right]\right]=0 .}
\end{gathered}
$$

These are the necessary and sufficient conditions for the consistency of the extended Lie algebra with generators $\hat{x}_{\lambda}$ and $M_{\mu \nu}$. It may be noted that (9), (10) have a smooth limit $\left[M_{\mu \nu}, x_{\lambda}\right]=x_{\mu} \delta_{\nu \lambda}-x_{\nu} \delta_{\mu \lambda}$, when $a_{\mu} \rightarrow 0$. There are infinitely many representations of $M_{\mu \nu}$ in terms of commutative coordinates $x_{\lambda}$, and their derivatives $\partial_{\lambda}$. If we require generators $M_{\mu \nu}$ to be linear in $x$ with an infinite series in $\partial$, then there emerge two families of solutions consistent simultaneously with the relations (8), (9) and (10). One family is for $\psi=1$ and the other is for $\psi=1+2 A$. Here we focus our attention only on the first family which gives us solutions of the form

$$
\begin{gathered}
M_{i j}=x_{i} \partial_{j}-x_{j} \partial_{i} \\
M_{i n}=x_{i} \partial_{n} \varphi \frac{e^{2 A}-1}{2 A}-x_{n} \partial_{i} \frac{1}{\varphi}+i a x_{i} \Delta \frac{1}{2 \varphi}-i a x_{k} \partial_{k} \partial_{i} \frac{\gamma}{\varphi} \\
M_{n i}=-M_{i n}
\end{gathered}
$$

where $\Delta=\partial_{k} \partial_{k}, \quad \gamma=\frac{\varphi^{\prime}}{\varphi}+1$ and the summation over repeated indices is understood. This realization can be parametrized by an arbitrary positive function $\varphi(A), \quad \varphi(0)=1$. To complete the settings we are dealing with, it is natural to introduce the Dirac derivatives $D_{\mu}$ and an invariant operator $\square$ as

$$
\begin{gathered}
{\left[M_{\mu \nu}, D_{\lambda}\right]=\delta_{\nu \lambda} D_{\mu}-\delta_{\mu \lambda} D_{\nu}} \\
{\left[D_{\mu}, D_{\nu}\right]=0 . \quad\left[M_{\mu \nu}, \square\right]=0, \quad\left[\square, \hat{x}_{\mu}\right]=2 D_{\mu} .}
\end{gathered}
$$

Realizations of the above operators in terms of the commutative variables have the form

$$
\begin{gathered}
D_{i}=\partial_{i} \frac{e^{-A}}{\varphi}, \\
D_{n}=\partial_{n} \frac{\sinh A}{A}+i a \Delta \frac{e^{-A}}{2 \varphi^{2}}, \\
\square=\Delta \frac{e^{-A}}{\varphi^{2}}-\partial_{n}^{2} \frac{2[1-\cosh A]}{A^{2}} .
\end{gathered}
$$

Similar expressions for $M_{\mu \nu}, D_{\lambda}$, as given in Eqs.(11)-(14), have been obtained in Refs. 20], 22], but only for three particular choices of the function $\varphi$, namely $\varphi=1, \varphi=e^{-A}$ and $\varphi=\frac{A}{e^{A}-1}$. Now we calculate the commutation relations between the $\mathrm{NC}$ coordinates $\hat{x}_{\mu}$ and the Dirac derivatives $D_{\nu}$ :

$$
\begin{gathered}
{\left[D_{i}, \hat{x}_{j}\right]=\delta_{i j}\left(-i a D_{n}+\sqrt{1-a^{2} D_{\mu} D_{\mu}}\right),} \\
{\left[D_{i}, \hat{x}_{n}\right]=0,} \\
{\left[D_{n}, \hat{x}_{i}\right]=i a D_{i},} \\
{\left[D_{n}, \hat{x}_{n}\right]=\sqrt{1-a^{2} D_{\mu} D_{\mu}} .}
\end{gathered}
$$

These relations are fixed by all previous commutation relations (3), (8), (19), (10), (12) and they involve only the deformation parameter $a$. 


\subsection{Lebniz rules and the coproducts}

An important ingredient of the symmetry structure of $\kappa$-deformed space are the Leibniz rules of generators of rotations and derivatives. For the case in consideration, the Leibniz rule for the rotation generators looks like

$$
\begin{gathered}
M_{i j}(\hat{f} \cdot \hat{g})=\left(M_{i j} \hat{f}\right) \cdot \hat{g}+\hat{f} \cdot\left(M_{i j} \hat{g}\right), \\
M_{i n}(\hat{f} \cdot \hat{g})=\left(M_{i n} \hat{f}\right) \cdot \hat{g}+\left(e^{A} \hat{f}\right) \cdot\left(M_{i n} \hat{g}\right)+i a\left(\partial_{j} \frac{1}{\varphi(A)} \hat{f}\right) \cdot\left(M_{i j} \hat{g}\right),
\end{gathered}
$$

where $\hat{f}, \hat{g}$ are functions of the NC coordinates $\hat{x}_{\mu}$. Similarly, we have the expressions

$$
\begin{aligned}
D_{n}(\hat{f} \cdot \hat{g})= & \left(D_{n} \hat{f}\right) \cdot\left(e^{-A} \hat{g}\right)+\left(\frac{i a D_{n}+\sqrt{1-a^{2} D_{\mu} D_{\mu}}}{1-a^{2} D_{k} D_{k}} \hat{f}\right) \cdot\left(D_{n} \hat{g}\right) \\
+ & \left(i a D_{i} \frac{i a D_{n}+\sqrt{1-a^{2} D_{\mu} D_{\mu}}}{1-a^{2} D_{k} D_{k}}\right) \cdot\left(D_{i} \hat{g}\right), \\
& D_{i}(\hat{f} \cdot \hat{g})=\left(D_{i} \hat{f}\right) \cdot\left(e^{-A} \hat{g}\right)+\hat{f} \cdot\left(D_{i} \hat{g}\right),
\end{aligned}
$$

as the Leibniz rules for the Dirac derivative.

The above equations for the Leibniz rules comprise the notion of coproducts. In the case of the rotation generators, the coproduct looks as

$$
\begin{gathered}
\triangle M_{i j}=M_{i j} \otimes 1+1 \otimes M_{i j}, \\
\triangle M_{i n}=M_{i n} \otimes 1+e^{A} \otimes M_{i n}+i a D_{j} e^{A} \otimes M_{i j},
\end{gathered}
$$

while, in the case of the Dirac derivative, it is given by

$$
\begin{gathered}
\triangle D_{n}=D_{n} \otimes\left(-i a D_{n}+\sqrt{1-a^{2} D_{\mu} D_{\mu}}\right)+\frac{i a D_{n}+\sqrt{1-a^{2} D_{\mu} D_{\mu}}}{1-a^{2} D_{k} D_{k}} \otimes D_{n} \\
+i a D_{i} \frac{i a D_{n}+\sqrt{1-a^{2} D_{\mu} D_{\mu}}}{1-a^{2} D_{k} D_{k}} \otimes D_{i}, \\
\triangle D_{i}=D_{i} \otimes\left(-i a D_{n}+\sqrt{1-a^{2} D_{\mu} D_{\mu}}\right)+1 \otimes D_{i},
\end{gathered}
$$

where $e^{A}$ is defined as

$$
e^{-A}=-i a D_{n}+\sqrt{1-a^{2} D_{\mu} D_{\mu}}
$$

The final result for the coproduct depends only on $a D_{n}$, and $a^{2} D_{\mu} D_{\mu}$. In the limit $a \rightarrow 0$, it gives the ordinary undeformed coproduct for $M_{\mu \nu}$, i.e. $D_{\mu}$, respectively. The notion of the coproduct leads to the observation that the generators of the $\kappa$-deformed symmetry are elements of a Hopf algebra. The coproduct $\triangle$, which we have determined for $M_{\mu \nu}$ and $D_{\mu}$, extends multiplicatively to the whole algebra $I S O_{a}(n)$, which becomes a Hopf algebra in this way. Some examples of the Poincaré invariant interpretation of NC spaces and of the twisted Poincaré coalgebra were also considered in [41], [42].

We note that from Eqs.(4) it follows that

$$
\left[\partial_{i}, \hat{x}_{j}\right]=\delta_{i j} \varphi(A) ; \quad\left[\partial_{i}, \hat{x}_{n}\right]=i a \partial_{i} \gamma(A)
$$




$$
\left[\partial_{n}, \hat{x}_{i}\right]=0 ; \quad\left[\partial_{n}, \hat{x}_{n}\right]=1
$$

The corresponding coproducts $\triangle_{\varphi}$ are

$$
\begin{gathered}
\triangle_{\varphi}\left(\partial_{n}\right)=\partial_{n} \otimes 1+1 \otimes \partial_{n}=\partial_{n}^{x}+\partial_{n}^{y}, \\
\triangle_{\varphi}\left(\partial_{i}\right)=\partial_{i}^{x} \frac{\varphi\left(A_{x}+A_{y}\right)}{\varphi\left(A_{x}\right)}+\partial_{i}^{y} \frac{\varphi\left(A_{x}+A_{y}\right)}{\varphi\left(A_{y}\right)} e^{A_{x}},
\end{gathered}
$$

where $A_{x}=i a \partial_{n}^{x}$ and similarly for $A_{y}$. They are consistent with Eqs.(16) and (13). At this point it is convenient to introduce a notion of natural realization, namely the $\varphi$ realizations (44) are natural with respect to Eqs.(17). The natural realization is generally characterized by the reqirement $D_{\mu} \equiv \partial_{\mu}$.

Particularly, for the deformed Heisenberg algebra (15), which is fixed in the case of the extended Lie algebra introduced in section 2.1, the natural realization is given by

$$
\hat{x}_{\mu}^{N a t}=x_{\mu} \sqrt{1-a^{2} \partial^{2}}-i C_{\alpha \mu \nu} \partial_{\alpha},
$$

with $C_{\alpha \mu \nu} \partial_{\alpha}=a_{\alpha} \delta_{\mu \nu}-a_{\mu} \delta_{\alpha \nu}$

\subsection{The correspondence of mapping and ordering}

In this section we want to establish the relation between different realizations of NC space and different ordering prescriptions. In other words, we want to make clear that for each mapping of the form (4) there exists an accompanying ordering prescripton. In the previous section we have introduced the natural realization which is seen as to not belong to the family (4). For this type of realization of $\mathrm{NC}$ space there also exists one particular ordering prescription. In this section we shall establish the general form of the orderings corresponding to the family of realizations (44), while in the section 4.2 , we shall deduce the form of the ordering prescription which belongs to the natural realization. To begin with, let us define the "vacuum" state

$$
\left|0>=1, \quad D_{\mu}\right| 0>=\partial_{\mu} \mid 0>=0,
$$

then for a given realization of $\mathrm{NC}$ space, described by $\varphi(A)$, there is a unique mapping from the set of fields $f(x)$ of the commutative coordinates $x_{\mu}$ to the set of fields $\hat{f}\left(\hat{x}_{\mu}\right)$ of the NC coordinates $\hat{x}_{\mu}$. This mapping $\Omega_{\varphi}$ can be characterized as

$$
\Omega_{\varphi}^{-1}: \hat{f}(\hat{x}) \longrightarrow f_{\varphi}(x) \text { such that } \hat{f}\left(\hat{x}_{\varphi}\right) \mid 0>=f_{\varphi}(x) .
$$

The fields or functions on NC space are conveniently introduced through the Fourier transform

$$
\hat{f}(\hat{x})=\int d^{n} x \tilde{f}_{\varphi}(k): e^{i k_{\alpha} \hat{x}_{\alpha}}:_{\varphi},
$$

where $::_{\varphi}$ denotes the ordering that corresponds to the realization (4) of NC space, parametrized by the function $\varphi$. The way in which this correspondence is realized will be made clear from the following consideration. Thus, to make a connection between the particular realization of NC space and the corresponding ordering prescription, we impose the requirement on the ordered exponentials

$$
: e^{i k \cdot \hat{x}_{\varphi}}: \varphi|0\rangle=e^{i k \cdot x}
$$

appearing in the expansion (22). For example, we have the following expressions for the left, right and symmetric ordering prescriptions, respectively:

$$
: e^{i k_{\alpha} \hat{x}_{\alpha}}:{ }_{L} \equiv e^{i k_{n} \hat{x}_{n}} e^{i k_{i} \hat{x}_{i}}=e^{i k_{n} \hat{x}_{n}+i k_{i} \hat{x}_{i} \varphi_{S}\left(-a k_{n}\right) e^{-a k_{n}}}
$$




$$
\begin{gathered}
: e^{i k_{\alpha} \hat{x}_{\alpha}}: R \equiv e^{i k_{i} \hat{x}_{i}} e^{i k_{n} \hat{x}_{n}}=e^{i k_{n} \hat{x}_{n}+i k_{i} \hat{x}_{i} \varphi_{S}\left(-a k_{n}\right)} \\
: e^{i k_{\alpha} \hat{x}_{\alpha}}: S=e^{i k_{\alpha} \hat{x}_{\alpha}}
\end{gathered}
$$

In the above relations we have introduced the function

$$
\varphi_{S}(A)=\frac{A}{e^{A}-1},
$$

where $A=i a \partial_{n} \equiv-a k_{n}$. Let us first consider the left ordered Fourier exponential. What we are interested here is to find the realization (4) for which this left ordered exponential will satisfy the requirement (23). Because all realizations are of the form (4), this basicaly reduces to the problem of finding the correct function $\varphi$. It can be easily shown that for the left ordering this function is $\varphi=e^{-A}$. By taking the same reasoning for the right ordered Fourier exponential, we end up with the function $\varphi=1$ as the one that defines the mapping (44) for which the right ordered exponential satisfies the requirement (23). In exactly the same way, the symmetric ordering is specified by the function $\varphi=\varphi_{S}$. There is also a family of ordering prescriptions, interpolating between left and right, namely,

$$
: e^{i k_{\alpha} \hat{x}_{\alpha}}: C=e^{i \lambda k_{n} \hat{x}_{n}} e^{i k_{i} \hat{x}_{i}} e^{i(1-\lambda) k_{n} \hat{x}_{n}}
$$

which corresponds to the realization characterized by the function $\varphi=e^{-\lambda A}$. For $\lambda=\frac{1}{2}$, this ordering prescription coincides with the one used in [29]. Generally, for the ordering prescription corresponding to the general mapping (44) with the function $\varphi$, we have

$$
: e^{i k_{\alpha} \hat{x}_{\alpha}}:_{\varphi}=e^{i k_{n} \hat{x}_{n}+i k_{i} \hat{x}_{i} \frac{\varphi_{S}\left(-a k_{n}\right)}{\varphi\left(-a k_{n}\right)}}
$$

It is clear that (27) incorporates all special cases (24) for the left, right and symmetric ordering, as well for the case (26), respectively.

Thus, the above consideration has shown that for each mapping, here specified by the function $\varphi$, there exists a particular basis $: e^{i k \cdot \hat{x}_{\varphi}}: \varphi$ of the ordered Fourier exponentials. That is, for each mapping there is a particular ordering prescription with the mutual correspondence between these two notions provided through the requirement (23). The aforementioned correspondence can be looked upon from a slightly different point of view. In respect to this, one can consider a more general form of the relation (23), namely,

$$
: e^{i k \cdot \hat{x}_{\varphi}}: \chi|0\rangle=e^{i K_{\varphi}^{\chi}(k) \cdot x}
$$

where $K_{\varphi}^{\chi}(k)$ is the function defined in (54) and has the momentum $k$ as an argument. In this expression, one can change realization of $\mathrm{NC}$ space as well as ordering prescription simply by changing the functions $\varphi$, $\chi$, respectively. By doing this, the requirement (23) will be fulfilled only after the changes in realization and ordering meet each other, i.e. it will be satisfied only when $\varphi=\chi$.

The relation (27) can be deduced from the $\mathrm{BCH}$ formula for the $\kappa$-deformed Lie algebra type of $\mathrm{NC}$ space

$$
e^{i k_{\alpha} \hat{x}_{\alpha}} e^{i q_{\alpha} \hat{x}_{\alpha}}=e^{i \mathcal{D}_{\alpha}(k, q) \hat{x}_{\alpha}}
$$

where

$$
\begin{gathered}
\mathcal{D}_{i}(k, q)=k_{i} \frac{\varphi_{S}\left(-a k_{n}-a q_{n}\right)}{\varphi_{S}\left(-a k_{n}\right)}+q_{i} \frac{\varphi_{S}\left(-a k_{n}-a q_{n}\right)}{\varphi_{S}\left(-a q_{n}\right)} e^{-a k_{n}}, \\
\mathcal{D}_{n}(k, q)=k_{n}+q_{n},
\end{gathered}
$$

and from the defining relation for the generalized momentum addition rule $k \oplus q$ which reads as

$$
: e^{i k_{\alpha} \hat{x}_{\alpha}}:_{\varphi}: e^{i q_{\alpha} \hat{x}_{\alpha}}:_{\varphi}=: e^{i(k \oplus q)_{\alpha} \hat{x}_{\alpha}}:_{\varphi}
$$


Inserting the relation (27) into the relation (31) and using (29), (30), we get

$$
\begin{gathered}
(k \oplus q)_{i}=k_{i} \frac{\varphi\left(-a k_{n}-a q_{n}\right)}{\varphi\left(-a k_{n}\right)}+q_{i} \frac{\varphi\left(-a k_{n}-a q_{n}\right)}{\varphi\left(-a q_{n}\right)} e^{-a k_{n}}, \\
(k \oplus q)_{n}=k_{n}+q_{n} .
\end{gathered}
$$

We see that the generalized momentum addition rule (32) is fully in agreement with the coproduct $\triangle_{\varphi}\left(\partial_{\mu}\right)$, obtained in the previous section, relation (18). Precisely, if we set $k=-i \partial_{1}, q=-i \partial_{2}$, then (18) and (32) can be related as $\triangle_{\varphi}(\partial)=\partial_{1} \oplus \partial_{2}$.

\subsection{T- operator}

Now we introduce a star product in a given $\varphi$-realization in the following way. As in the previous section, we define the "vacuum" state as

$$
\left|0>=1, \quad D_{\mu}\right| 0>=\partial_{\mu} \mid 0>=0 .
$$

and introduce the unique map $\Omega_{\varphi}$ which, for a given realization described by $\varphi(A)$, maps the commutative algebra of functions $f(x)$ into the noncommutative algebra of functions $\hat{f}\left(\hat{x}_{\mu}\right)$. The map $\Omega_{\varphi}$ can be uniquely characterized by

$$
\Omega_{\varphi}: f(x) \longrightarrow \hat{f}\left(\hat{x}_{\varphi}\right) \text { such that } \hat{f}\left(\hat{x}_{\varphi}\right) \mid 0>=f(x) .
$$

If we now have two functions $\hat{f}(\hat{x}), \hat{g}(\hat{x})$ of NC coordinates, make their product $\hat{f}(\hat{x}) \cdot \hat{g}(\hat{x})$ and ask which function of commutative coordinates this combination belongs to (through the mapping $\Omega_{\varphi}$ ), we arrive at

$$
\left(f \star_{\varphi} g\right)(x)=\hat{f}\left(\hat{x}_{\varphi}\right) \hat{g}\left(\hat{x}_{\varphi}\right) \mid 0>=\hat{f}\left(\hat{x}_{\varphi}\right) g(x) .
$$

By this expression the star product in a given $\varphi$ realization is defined. This product obviously depends on the realization $\varphi$. Generally, the star products belonging to two different realizations $\varphi_{1}$ and $\varphi_{2}$ can be related to each other by the so-called T- operator that identifies a class of equivalent star products. Thus, the T- operator can be characterized by (see for example Ref. 22])

$$
T\left(f(x) \star_{\varphi_{1}} g(x)\right)=T(f(x)) \star_{\varphi_{2}} T(g(x)) .
$$

In Ref. 22] $\mathrm{T}$ operator has been obtained by relating the cases for $\varphi=e^{-A}$ and $\varphi=1$ to that for $\varphi=\frac{A}{e^{A}-1}$, corresponding to left, right and symmetric ordering, respectively. To deduce its explicit form for the case of $\kappa$ deformed spaces, we take advantage of the following identities:

$$
\begin{gathered}
x_{i} \star_{\varphi} f(x)=\left(\hat{x}_{\varphi}\right)_{i} f(x)=x_{i} \varphi(A) f(x), \\
x_{n} \star_{\varphi} f(x)=\left(\hat{x}_{\varphi}\right)_{n} f(x)=\left[x_{n}+i a x_{k} \partial_{k} \gamma(A)\right] f(x),
\end{gathered}
$$

together with the obvious property $T\left(x_{i}\right)=x_{i}$ and $T\left(x_{n}\right)=x_{n}$. We can now proceed as follows:

$$
T\left(x_{i} \star_{\varphi_{1}} f(x)\right)=T\left(x_{i}\right) \star_{\varphi_{2}} T(f(x)) .
$$

By using (37), we have

$$
T\left(x_{i} \varphi_{1}(A) f(x)\right)=x_{i} \varphi_{2}(A) T(f(x))
$$

implying

$$
\left(x_{i} T+\frac{\partial T}{\partial \partial_{i}}\right) \varphi_{1}(A) f(x)=x_{i} \varphi_{2}(A) T f(x)
$$


Since (40) holds for any function $f(x)$, it reduces to

$$
x_{i}\left(\varphi_{1}(A)-\varphi_{2}(A)\right) T=-\frac{\partial T}{\partial \partial_{i}} \varphi_{1}(A),
$$

which integrates to

$$
T=\lim _{y \rightarrow x} e^{x_{i} \partial_{i}^{y}\left(\frac{\varphi_{2}(A)}{\varphi_{1}(A)}-1\right)} \equiv: e^{x_{i} \partial_{i}\left(\frac{\varphi_{2}(A)}{\varphi_{1}(A)}-1\right)}:,
$$

where the symbol :: has the meaning of the usual normal ordering with the variables $x_{i}$ coming to the left with respect to the variables $\partial_{i}$ and should not be confused with the $::_{\varphi}$ used before. The solution (42) also satisfies the equation

$$
\frac{\partial T}{\partial \partial_{n}}=\operatorname{iax}_{k} \partial_{k}\left(\gamma_{2}(A)-\gamma_{1}(A)\right) T
$$

stemming from the condition

$$
T\left(x_{n} \star_{\varphi_{1}} f(x)\right)=T\left(x_{n}\right) \star_{\varphi_{2}} T(f(x)) .
$$

Using the formula (A. 5) from Appendix A, we can easily find the inverse of (42) which reads

$$
T^{-1}=: e^{x_{i} \partial_{i}\left(\frac{\varphi_{1}(A)}{\varphi_{2}(A)}-1\right)}: .
$$

If we introduce the notation $T_{1,2} \equiv T=: e^{x_{i} \partial_{i}\left(\frac{\varphi_{1}(A)}{\varphi_{2}(A)}-1\right)}:$, we can summarize the properties of the $T-$ operator as follows:

1. inverse operator,

$$
T_{1,2}^{-1}=T_{2,1}
$$

2. transitivity,

$$
T_{1,2} T_{2,3}=T_{1,3} .
$$

Finally, using Eq.(A. 17) in Appendix A, we can write the operator T, Eq.(42), as

$$
T=e^{x_{i} \partial_{i} \ln \frac{\varphi_{2}}{\varphi_{1}}} .
$$

It is important to note that we can represent $\hat{x}$ in terms of two representations $\varphi_{1}(A)$ and $\varphi_{2}(A)$ as

$$
\begin{aligned}
\hat{x}_{i}=\left(x_{1}\right)_{i} \varphi_{1}(A) & =\left(x_{2}\right)_{i} \varphi_{2}(A), \\
\hat{x}_{n}=\left(x_{1}\right)_{n}+i a\left(x_{1}\right)_{k}\left(\partial_{1}\right)_{k} \gamma_{1}(A) & =\left(x_{2}\right)_{n}+i a\left(x_{2}\right)_{k}\left(\partial_{2}\right)_{k} \gamma_{2}(A),
\end{aligned}
$$

where for the transformation of the coordinates we have

$$
\begin{gathered}
\left(x_{2}\right)_{i}=T^{-1}\left(x_{1}\right)_{i} T=\left(x_{1}\right)_{i} \frac{\varphi_{1}(A)}{\varphi_{2}(A)}, \\
\left(x_{2}\right)_{n}=T^{-1}\left(x_{1}\right)_{n} T=\left(x_{1}\right)_{n}+i a\left(x_{1}\right)_{k}\left(\partial_{1}\right)_{k}\left(\gamma_{1}(A)-\gamma_{2}(A)\right) .
\end{gathered}
$$

Similarly, for the transformation of the derivatives, it follows

$$
\begin{gathered}
\left(\partial_{2}\right)_{i}=T^{-1}\left(\partial_{1}\right)_{i} T=\left(\partial_{1}\right)_{i} \frac{\varphi_{2}(A)}{\varphi_{1}(A)}, \\
\left(\partial_{2}\right)_{n}=T^{-1}\left(\partial_{1}\right)_{n} T=\left(\partial_{1}\right)_{n} .
\end{gathered}
$$

Generally, one can introduce a mapping $K_{\varphi_{2}}^{\varphi_{1}}: R^{n} \longrightarrow R^{n}$, transforming the vector $\partial_{\varphi_{1}}$ into vector $\partial_{\varphi_{2}}$,

$$
\partial_{\varphi_{2}}=T^{-1} \partial_{\varphi_{1}} T=K_{\varphi_{2}}^{\varphi_{1}}\left(\partial_{\varphi_{1}}\right)
$$

Because of the transitivity property of the $T$-operator, the composition of $K$ functions satisfies the relation

$$
K_{\varphi_{2}}^{\varphi_{1}} K_{\varphi_{3}}^{\varphi_{2}}=K_{\varphi_{3}}^{\varphi_{1}} .
$$




\section{DERIVATION OF THE STAR PRODUCT CORRESPONDING TO THE ARBITRARY ORDERING PRESCRIPTON / MAPPING}

We now proceed in some detail to find the star product for $\kappa$-deformed space, corresponding to the particular ordering $::_{\varphi}$. It can be deduced from the defining relation (36), starting from the expression for the left star product, which is known from the literature as

$$
f(x) \star_{L} g(x)=\lim _{\substack{y \rightarrow x \\ z \rightarrow x}} e^{x_{i} \partial_{i}^{y}\left(e^{-i a \partial_{n}^{z}}-1\right)} f(y) g(z)=\lim _{y \rightarrow x} e^{-i a x_{i} \partial_{i}^{x} \partial_{n}^{y}} f(x) g(y) .
$$

Thus, from $T\left(f(x) \star_{L} g(x)\right)=T(f(x)) \star_{\varphi} T(g(x))$, it follows

$$
\left(\tilde{f} \star_{\varphi} \tilde{g}\right)(x)=T\left(T^{-1} \tilde{f} \star_{L} T^{-1} \tilde{g}\right),
$$

where $\tilde{f}=T f$ is introduced. For simplicity, in due course, we drop the symbol $\sim$ from the functions and by using the relations (42), (45) and (56), we arrive at

$$
\begin{gathered}
\left(f \star_{\varphi} g\right)(x)=\lim _{w \rightarrow x} e^{x_{j} \partial_{j}^{w}\left(\varphi\left(A_{w}\right) e^{A_{w}}-1\right)} \lim _{\substack{z \rightarrow w \\
y \rightarrow w}} e^{w_{j} \partial_{j}^{z}\left(e^{-A_{y}}-1\right)} \times \\
\times \lim _{u \rightarrow z} e^{z_{j} \partial_{j}^{u}\left(\frac{e^{-A_{u}}}{\varphi\left(A_{u}\right)}-1\right)} \lim _{t \rightarrow y} e^{y_{j} \partial_{j}^{t}\left(\frac{e^{-A_{t}}}{\varphi\left(A_{t}\right)}-1\right)} f(u) g(t),
\end{gathered}
$$

with the abbreviation $A_{x}=i a \frac{\partial}{\partial x_{n}}$, etc. The second and the third term in (58) can be contracted with the help of Eq. (A. 5 to yield

$$
\begin{gathered}
\left(f \star_{\varphi} g\right)(x)=\lim _{w \rightarrow x} e^{x_{j} \partial_{j}^{w}\left(\varphi\left(A_{w}\right) e^{A_{w}}-1\right)}: e^{z_{j} \partial_{j}^{z}\left(e^{-A_{y}}-1\right)}:: e^{z_{j} \partial_{j}^{z}\left(\frac{e^{-A_{u}}}{\varphi\left(A_{u}\right)}-1\right)}: \\
\lim _{t \rightarrow y} e^{y_{j} \partial_{j}^{t}\left(\frac{e^{-A_{t}}}{\varphi\left(A_{t}\right)}-1\right)} f(u) g(t) \\
=\lim _{w \rightarrow x} e^{x_{j} \partial_{j}^{w}\left(\varphi\left(A_{w}\right) e^{A_{w}}-1\right)}: e^{N\left(e^{-A_{y}} \frac{e^{-A_{u}}}{\varphi\left(A_{u}\right)}-1\right)}: \lim _{t \rightarrow y} e^{y_{j} \partial_{j}^{t}\left(\frac{e^{-A_{t}}}{\varphi\left(A_{t}\right)}-1\right)} f(u) g(t) \\
=\lim _{w \rightarrow x} e^{x_{j} \partial_{j}^{w}\left(\varphi\left(A_{w}\right) e^{A_{w}}-1\right)} \lim _{\substack{u \rightarrow w \\
y \rightarrow w}} e^{w_{j} \partial_{j}^{u}\left(e^{-A_{y}} \frac{e^{-A_{u}}}{\varphi\left(A_{u}\right)}-1\right)} \lim _{t \rightarrow y} e^{y_{j} \partial_{j}^{t}\left(\frac{e^{-A_{t}}}{\varphi\left(A_{t}\right)}-1\right)} f(u) g(t) .
\end{gathered}
$$

If the obvious relation $\varphi\left(A_{w}\right) \lim _{t \rightarrow y} f(t)=\lim _{t \rightarrow y} \varphi\left(A_{t}\right) f(t)$ and the similar one (B. 1) derived in Appendix B are applied to the second and the third factor in (60), we get

$$
\begin{aligned}
& \left(f \star_{\varphi} g\right)(x)=\lim _{w \rightarrow x} e^{x_{j} \partial_{j}^{w}\left(\varphi\left(A_{w}\right) e^{A_{w}}-1\right)} \lim _{\substack{u \rightarrow w \\
t \rightarrow w}} e^{w_{j} \partial_{j}^{u}\left(e^{-A_{t}} \frac{e^{-A_{u}}}{\varphi\left(A_{u}\right)}-1\right)} e^{w_{j} \partial_{j}^{t}\left(\frac{e^{-A_{t}}}{\varphi\left(A_{t}\right)}-1\right)} f(u) g(t) \\
& =\lim _{w \rightarrow x} \lim _{\substack{u \rightarrow w \\
t \rightarrow w}} e^{x_{j}\left(\partial_{j}^{u}+\partial_{j}^{t}\right)\left(\varphi\left(A_{u}+A_{t}\right) e^{A_{u}+A_{t}}-1\right)} e^{w_{j} \partial_{j}^{u}\left(e^{-A_{t}} \frac{e^{-A_{u}}}{\varphi\left(A_{u}\right)}-1\right)} e^{w_{j} \partial_{j}^{t}\left(\frac{e^{-A_{t}}}{\varphi\left(A_{t}\right)}-1\right)} f(u) g(t) .
\end{aligned}
$$

Here we can again use the equivalent representation of the factors in (61) through the normal ordering (not to be confused with :: $:_{\varphi}$ and apply (A. 5) to obtain

$$
\begin{gathered}
\left(f \star_{\varphi} g\right)(x)=\left(\lim _{\substack{u \rightarrow x \\
t \rightarrow x}} e^{x_{j} \partial_{j}^{u}\left(\varphi\left(A_{u}+A_{t}\right) e^{A_{u}+A_{t}}-1\right)} e^{x_{j} \partial_{j}^{u}\left(e^{-A_{t}} \frac{e^{-A_{u}}}{\varphi\left(A_{u}\right)}-1\right)}\right)\left(\lim _{\substack{u \rightarrow x \\
t \rightarrow x}} e^{x_{j} \partial_{j}^{t}\left(\varphi\left(A_{u}+A_{t}\right) e^{A_{u}+A_{t}}-1\right)} e^{x_{j} \partial_{j}^{t}\left(\frac{e^{-A_{t}}}{\varphi\left(A_{t}\right)}-1\right)}\right) f(u) g(t) \\
=\left(: e^{N\left(\varphi\left(A_{u}+A_{t}\right) e^{A_{u}+A_{t}}-1\right)}:: e^{N\left(e^{-A_{t}} \frac{e^{-A_{u}}}{\varphi\left(A_{u}\right)}-1\right)}:\right)\left(: e^{N\left(\varphi\left(A_{u}+A_{t}\right) e^{A_{u}+A_{t}}-1\right)}:: e^{N\left(\frac{e^{-A_{t}}}{\varphi\left(A_{t}\right)}-1\right)}:\right) f(u) g(t)
\end{gathered}
$$




$$
\begin{gathered}
=: e^{N\left(\varphi\left(A_{u}+A_{t}\right) e^{A_{u}+A_{t}} e^{-A_{t}} \frac{e^{-A_{u}}}{\varphi\left(A_{u}\right)}-1\right)}:: e^{N\left(\varphi\left(A_{u}+A_{t}\right) e^{A_{u}+A_{t}} \frac{e^{-A_{t}}}{\varphi\left(A_{t}\right)}-1\right)}: f(u) g(t) \\
=: e^{N\left(\frac{\varphi\left(A_{u}+A_{t}\right)}{\varphi\left(A_{u}\right)}-1\right)}:: e^{N\left(\frac{\varphi\left(A_{u}+A_{t}\right)}{\varphi\left(A_{t}\right)} e^{A_{u}}-1\right)}: f(u) g(t)=\lim _{u \rightarrow x} e^{x_{j} \partial_{j}^{u}\left(\frac{\varphi\left(A_{u}+A_{t}\right)}{\varphi\left(A_{u}\right)}-1\right)} \lim _{t \rightarrow x} e^{x_{j} \partial_{j}^{t}\left(\frac{\varphi\left(A_{u}+A_{t}\right)}{\varphi\left(A_{t}\right)} e^{A_{u}}-1\right)} f(u) g(t) .
\end{gathered}
$$

Since the factors in (62) commute, we finally obtain the star product in the $\varphi$ realization (i.e. in the $\varphi$ ordering)

$$
\left(f \star_{\varphi} g\right)(x)=\lim _{\substack{u \rightarrow x \\ t \rightarrow x}} e^{x_{j} \partial_{j}^{u}\left(\frac{\varphi\left(A_{u}+A_{t}\right)}{\varphi\left(A_{u}\right)}-1\right)+x_{j} \partial_{j}^{t}\left(\frac{\varphi\left(A_{u}+A_{t}\right)}{\varphi\left(A_{t}\right)} e^{A_{u}}-1\right)} f(u) g(t) .
$$

It should be noted that the coproduct stemming from this result for the star product completely coincides with the relation (32) and the coproduct (18). We stress here that the result (63) is the special case of the more general one

$$
(f \star g)(x)=\lim _{\substack{u \rightarrow x \\ y \rightarrow x}} \mu\left(e^{x_{\alpha}\left(\triangle-\triangle_{0}\right) \partial_{\alpha}} f(u) \otimes g(y)\right),
$$

where $\triangle_{0}\left(\partial_{\mu}\right)=\partial_{\mu} \otimes 1+1 \otimes \partial_{\mu}, \triangle\left(\partial_{\mu}\right)$ is given in (18) and $\mu$ is the multiplication map in the Hopf algebra, namely, $\mu(f(x) \otimes g(x))=f(x) g(x)$. The relation (64) can be extended to hold for a general Lie algebra. To end this section, note that generally, in view of Eqs.(31) and (35), the star product $\star_{\varphi}$ satisfies the relation $e^{i k \cdot x_{\varphi}} e^{i q \cdot x}=e^{i(k \oplus q) \cdot x}$, with $k \oplus q$ defined in (32).

\section{TOWARDS THE SCALAR FIELD THEORY ON $\kappa$-DEFORMED SPACE}

\section{1. $\quad \kappa$-deformed Minkowski space and the natural realization}

All results obtained so far are derived for the case of the Euclidean metric. They can be easily extended to Minkowski space. It is only about the change in the definition of the momentum

$$
P_{0}=i \partial_{0}, \quad P_{i}=-i \partial_{i}, \quad i=1,2, \ldots, N-1
$$

in order to satisfy

$$
\left[P_{\mu}, x_{\nu}\right]=-i \eta_{\mu \nu}, \quad \mu, \nu=0,1, \ldots, N-1
$$

$\eta_{\mu \nu}=\operatorname{diag}(-1,+1,+1,+1)$, while the algebra of the variables $x_{\mu}, \partial_{\nu}$ remains unaltered,

$$
\left[\partial_{\mu}, x_{\nu}\right]=\delta_{\mu \nu}, \quad \mu, \nu=0,1, \ldots, N-1
$$

Accordingly, the commutation relation describing $\kappa$-deformed Minkowski space casts into

$$
\left[\hat{x}_{i}, \hat{x}_{j}\right]=0, \quad\left[\hat{x}_{0}, \hat{x}_{i}\right]=i a \hat{x}_{i}, \quad i, j=1,2, \ldots, N-1 .
$$

The star product (63) on $\kappa$-deformed Minkowski space also remains unchanged, except that $A_{x}$ now stands for $A_{x}=i a \partial_{0}^{x}$. It is important to stress that the deformation parameter $a$ appearing in the above commutation relation is not the same as the one we were dealing with when we considered the Euclidean space. They are related as $a_{n}=i a_{0}$, where $a_{n}$ is the old deformation parameter and $a_{0}$ is the new one. For simplicity, we remove the index 0 of it, but in all subsequent consideration one should keep in mind that it is the time component of the $n$-vector in Minkowski space and that it is real. The interchange $a_{n}=i a_{0}$, together with the whole set of relations $x_{n}=i x_{0}, D_{n}=-i D_{0}, M_{i n}=-i M_{i 0}$ provides the consistent transition from Euclidean to Minkowski space.

Among all mappings that realize $\kappa$-deformed Minkowski space, there is one of special significance because, unlike the general mapping (4), it is covariant. It has some interesting properties and is introduced in a natural way by identifying the Dirac covariant derivative with the ordinary derivative $D_{\mu} \equiv \partial_{\mu}$. This special kind of realization has already been introduced at the end of section 2.2 through the mapping (19) to which we have assigned the term 
natural realization. Since we are interested in this kind of realization with respect to the deformed Heisenberg algebra (15), according to (15), we have

$$
\left[\partial_{\mu}, \hat{x}_{\nu}^{N a t}\right]=\delta_{\mu \nu}\left(-i a \partial_{0}+\sqrt{1+a^{2} \partial^{2}}\right)+i a \delta_{\mu 0} \partial_{\nu}
$$

yielding the following realization of the noncommuting coordinates:

$$
\hat{x}_{\mu}^{N a t}=x_{\mu}\left(-i a \partial_{0}+\sqrt{1+a^{2} \partial^{2}}\right)+i a x_{0} \partial_{\mu} .
$$

Thus, in the following (70) will be referred to as a natural realization and it will be understood that it is natural with respect to the deformed Heisenberg algebra (15). We recall that (15) is fixed since it is a part of the extended Lorentz algebra (see section 2.1). Note that the mapping (70) is not incorporated in the set of realizations (4). The properties of the natural realization are expressed in a most convenient way if the Dirac covariant derivative (13) is associated with the momentum $P_{\mu}^{\varphi}$ on $\kappa$-deformed Minkowski space,

$$
\begin{gathered}
P_{i}^{\varphi}=-i D_{i}=-i \partial_{i} \frac{e^{-A}}{\varphi(A)}=k_{i} \frac{e^{-a k_{0}}}{\varphi\left(a k_{0}\right)} \\
P_{0}^{\varphi}=i D_{0}=i \partial_{0} \frac{\sinh A}{A}+a \Delta \frac{e^{-A}}{2 \varphi^{2}}=\frac{1}{a} \sinh \left(a k_{0}\right)-a \vec{k}^{2} \frac{e^{-a k_{0}}}{2 \varphi^{2}}
\end{gathered}
$$

where $k_{0}=i \partial_{0}, \quad k_{i}=-i \partial_{i}$. This association can be regarded as a map between the algebra of fields on noncommutative $\kappa$-Minkowski space and the algebra of ordinary fields on Minkowski spacetime equipped with a star product [24], 25]. The special case when $\varphi=1$, which is the case of right ordering, was considered in [30]. Since the map (71) is fully specified by its action on plane waves, it can be looked on as if it mapped ordinary Minkowski plane waves $e^{i k_{\alpha} x^{\alpha}}$ to plane waves $: e^{i P_{\alpha}(k) \hat{x}^{\alpha}}:_{\varphi}$ on $\kappa$-deformed Minkowski space, $P: k \mapsto P(k)$, with $P(k)$ given by (71).

The natural realization has a property

$$
e^{i k^{\alpha} \hat{x}_{\alpha}^{N a t}}|0\rangle=e^{i P_{\alpha}^{S}(k) x^{\alpha}},
$$

where $P_{\alpha}^{S}$ is the momentum (71) evaluated for $\varphi=\varphi_{S}$, i.e. corresponding to symmetric ordering. From this and the relations (71) it is easy to calculate the following expression:

$$
: e^{i k^{\alpha} \hat{x}_{\alpha}^{N a t}}:_{\varphi}|0\rangle=e^{i\left(-k_{0} \hat{x}_{0}^{N a t}+k_{i} \hat{x}_{i}^{N a t} \frac{\varphi_{S}\left(a k_{0}\right)}{\varphi\left(a k_{0}\right)}\right)}|0\rangle \equiv e^{i K_{\varphi}^{\alpha}(k) \hat{x}_{\alpha}^{N a t}}|0\rangle=e^{i P^{\varphi, \alpha}(k) x_{\alpha}},
$$

with $K_{\varphi}(k)=\left(k_{0}, k_{i} \frac{\varphi_{S}\left(a k_{0}\right)}{\varphi\left(a k_{0}\right)}\right)$ and $P_{\alpha}^{\varphi}$ given in (71). The function $K_{\varphi}(k)$ is an example of the map introduced in (54).

\subsection{Free scalar field theory on $\kappa$-deformed Minkowski space}

The natural realization appears as a particularly convenient one for constructing the field theory on noncommutative space. The reason for this is that it enables one to recast the action belonging to an algebra of functions whose product is a noncommutative star product into the action expressed entirely in terms of usual pointwise multiplication between two functions. We shall see that although the construction that is to be made requires one particular noncommutative star product on an algebra of functions, namely the one which is related to the natural realization, the realization of the NC space alone is not important at all. In other words, the realizations of 4-momenta and coordinates on NC space are not important in constructing the noncommutative field theory action. The final result, obtained from the original action by virtue of the properties of natural star product $\star_{N}$, will be cast into the form of the nonlocal, relativistically invariant field theory whose action is expressed in terms of usual pointwise multiplication of fields and has particularly simple form. This result is the same regardless of the particular realization used to represent 4-momenta and coordinates on NC space.

So far we have seen that each realization of $\mathrm{NC}$ space was related to some specific type of the ordering prescription. The same situation as well appears to be true in the case of the natural realization. As was the case with the 
realizations (4), where the corresponding orderings are expressed in terms of the exponentials of the type

$$
: e^{i k^{\alpha} \hat{x}_{\alpha}}:_{\varphi}=e^{-i k_{0} \hat{x}_{0}+i k_{i} \hat{x}_{i} \frac{\varphi S\left(a k_{0}\right)}{\varphi\left(a k_{0}\right)}}
$$

(The relation (74) is the same as (27), the difference being only in the signature adjusted to hold for the Minkowski space) the similar connection can be found for the natural realization either. In fact, it can immediately be deduced from Eq.(72) by rearranging it to obtain

$$
e^{i\left(P^{S}\right)^{-1}(k) \cdot \hat{x}^{N a t}}|0\rangle=e^{i k \cdot x}
$$

In order to specify the function $\left(P^{S}\right)^{-1}$, appearing in (75), we once again write the relations (71),

$$
\begin{gathered}
P_{i}^{\varphi}=k_{i} \frac{e^{-a k_{0}}}{\varphi\left(a k_{0}\right)}, \\
P_{0}^{\varphi}=\frac{1}{a} \sinh \left(a k_{0}\right)-a \vec{k}^{2} \frac{e^{-a k_{0}}}{2 \varphi^{2}\left(a k_{0}\right)} .
\end{gathered}
$$

By inverting them for the case when $\varphi=\varphi_{S}$, we obtain the required quantity $\left(P^{S}\right)^{-1}$ in components

$$
\begin{gathered}
\left(P^{S}\right)_{0}^{-1}(P)=\frac{1}{a} \ln \frac{a P_{0}+\sqrt{1-a^{2} P^{2}}}{1-a^{2} \bar{P}^{2}} \\
\left(P^{S}\right)_{i}^{-1}(P)=P_{i} \varphi_{S}\left(\ln \frac{a P_{0}+\sqrt{1-a^{2} P^{2}}}{1-a^{2} \bar{P}^{2}}\right) \frac{a P_{0}+\sqrt{1-a^{2} P^{2}}}{1-a^{2} \vec{P}^{2}} .
\end{gathered}
$$

The expression (75), together with (77), defines the ordering prescription corresponding to the natural realization.

We now turn to the notion of the star product corresponding to the natural realization. This star product, for which we adopt the designation $\star_{N}$, is characterized by the property

$$
e^{i P^{\varphi}(k) \cdot x} \star_{N} e^{i P^{\varphi}(q) \cdot x}=e^{i P^{\varphi}(k \oplus q) \cdot x},
$$

where

$$
k \oplus q=\left(k_{0}+q_{0}, \quad k_{i} \frac{\varphi\left(a k_{0}+a q_{0}\right)}{\varphi\left(a k_{0}\right)}+q_{i} \frac{\varphi\left(a k_{0}+a q_{0}\right)}{\varphi\left(a q_{0}\right)} e^{a k_{0}}\right)
$$

is the generalized rule for the addition of momenta and $P^{\varphi}$ is defined in (71). It is understood that the quantity $k \oplus q$ is taken in the $\varphi$ realization, although it is not specificaly indicated. The relation (78) will have a crucial role in our construction of noncommutative field theory. From the definition of the inverse 4-momentum $k \oplus k^{-1}=(0, \overrightarrow{0})$, if $k=\left(k_{0}, \vec{k}\right)$, then we have

$$
\begin{gathered}
k^{-1}=\left(-k_{0},-k_{i} \frac{\varphi\left(-a k_{0}\right)}{\varphi\left(a k_{0}\right)} e^{-a k_{0}}\right), \\
k^{-1} \oplus q=\left(-k_{0}+q_{0}, \quad\left(-\frac{k_{i}}{\varphi\left(a k_{0}\right)}+\frac{q_{i}}{\varphi\left(a q_{0}\right)}\right) \varphi\left(-a k_{0}+a q_{0}\right) e^{-a k_{0}}\right) .
\end{gathered}
$$

In the calculations that follow, we adopt the Hermitean conjugation property

$$
\left(e^{i P^{\varphi}(k) \cdot x}\right)^{\dagger}=e^{i P^{\varphi}\left(k^{-1}\right) \cdot x}
$$

Let us now derive the relation (78) which encloses the property of natural star product important for our construction. Utilizing Eqs.(31) and (73), we have

$$
\begin{gathered}
e^{i P^{\varphi}(k) \cdot x} \star_{N} e^{i P^{\varphi}(q) \cdot x} \\
=: e^{i k \cdot \hat{x}^{N a t}}:_{\varphi}: e^{i q \cdot \hat{x}^{N a t}}:_{\varphi}|0\rangle
\end{gathered}
$$




$$
\begin{gathered}
=: e^{i(k \oplus q) \cdot \hat{x}^{N a t}}: \varphi|0\rangle \\
=e^{i P^{\varphi}(k \oplus q) \cdot x},
\end{gathered}
$$

thus completing the proof of (78). In the second line of the derivation (83), we have applied Eq.(73), while in the third line we have used the property (31) satisfied by the generalized addition of momenta (adjusted for the Minkowski signature). Finaly, in the last line, Eq.(73) is used again, but in a reverse direction.

The result (78) can be further used to derive the identity

$$
\int d^{n} x \phi^{\dagger}(x) \star_{N} \psi(x)=\int d^{n} x \phi^{*}(x) \sqrt{1+a^{2} \partial_{\mu} \partial^{\mu}} \psi(x),
$$

which provides the transition from the integration of the star product of two fields to the integration of the ordinary product of fields. To prove it, it is sufficient to show that it holds for plane waves $\phi(x)=e^{i P^{\varphi}(k) \cdot x}, \psi(x)=e^{i P^{\varphi}(q) \cdot x}$. Thus, from (78) and (82) we have

$$
\int d^{n} x\left(e^{i P^{\varphi}(k) \cdot x}\right)^{\dagger} \star_{N} e^{i P^{\varphi}(q) \cdot x}=\int d^{n} x e^{i P^{\varphi}\left(k^{-1} \oplus q\right) \cdot x}=(2 \pi)^{n} \delta\left(P_{0}^{\varphi}\left(k^{-1} \oplus q\right)\right) \delta^{(n-1)}\left(\vec{P}^{\varphi}\left(k^{-1} \oplus q\right)\right) .
$$

In order to perform the calculations on the right-hand side of (85), it is convenient to introduce the quantities

$$
\begin{gathered}
\mathcal{P}_{n}^{\varphi}(k)=-\frac{1}{a} \cosh \left(a k_{0}\right)+a \vec{k}^{2} \frac{e^{-a k_{0}}}{2 \varphi^{2}\left(a k_{0}\right)}, \\
P_{ \pm}^{\varphi}=\mathcal{P}_{n}^{\varphi} \pm P_{0}^{\varphi}
\end{gathered}
$$

(The first quantity in (86) should not be confused with the Euclidean counterpart $P_{n}=i P_{0}$ of $P_{0}$.) It is now straightforward to obtain the following expressions:

$$
\begin{gathered}
P_{i}^{\varphi}\left(k^{-1} \oplus q\right)=\left(\frac{q_{i}}{\varphi\left(a q_{0}\right)}-\frac{k_{i}}{\varphi\left(a k_{0}\right)}\right) e^{-a q_{0}}, \\
P_{0}^{\varphi}\left(k^{-1} \oplus q\right)=\frac{1}{a} \sinh \left(a q_{0}-a k_{0}\right)-\frac{a}{2}\left(\frac{\vec{q}}{\varphi\left(a q_{0}\right)}-\frac{\vec{k}}{\varphi\left(a k_{0}\right)}\right)^{2} e^{-a k_{0}-a q_{0}}, \\
P_{+}^{\varphi}(k)=-\frac{1}{a} e^{-a k_{0}} .
\end{gathered}
$$

When they are combined with (76), we get

$$
\begin{gathered}
P_{i}^{\varphi}\left(k^{-1} \oplus q\right)=\frac{P_{+}^{\varphi}(k) P_{i}^{\varphi}(q)-P_{i}^{\varphi}(k) P_{+}^{\varphi}(q)}{P_{+}^{\varphi}(k)} \\
P_{0}^{\varphi}\left(k^{-1} \oplus q\right)=-a\left[P_{+}^{\varphi}(k) P_{0}^{\varphi}(q)-P_{0}^{\varphi}(k) P_{+}^{\varphi}(q)-P_{i}^{\varphi}(k) P_{i}^{\varphi}\left(k^{-1} \oplus q\right)\right] .
\end{gathered}
$$

Since the expression (85) vanishes for $\vec{P}^{\varphi}\left(k^{-1} \oplus q\right) \neq 0$, the last term of $P_{0}^{\varphi}\left(k^{-1} \oplus q\right)$ in (88) drops out and we are left with

$$
P_{+}^{\varphi}(k) P_{0}^{\varphi}(q)-P_{0}^{\varphi}(k) P_{+}^{\varphi}(q)=\frac{1}{2 a^{2} P_{+}^{\varphi}(k) P_{+}^{\varphi}(q)}\left[P_{+}^{\varphi}(q)^{2}-P_{+}^{\varphi}(k)^{2}+a^{2}\left(P_{+}^{\varphi}(k) \vec{P}^{\varphi}(q)\right)^{2}-a^{2}\left(P_{+}^{\varphi}(q) \vec{P}^{\varphi}(k)\right)^{2}\right]
$$

where use has been made of the identity $2 P_{0}^{\varphi}=P_{+}^{\varphi}-\frac{1}{a^{2} P_{+}^{\varphi}}+\frac{\vec{P}_{\varphi}^{2}}{P_{+}^{\varphi}}$. From the same argument as before, the last two terms in (89) cancel each other, leaving us with

$$
\begin{gathered}
(2 \pi)^{n} \delta^{(n)}\left(P^{\varphi}\left(k^{-1} \oplus q\right)\right)=(2 \pi)^{n} \delta\left(-a \frac{P_{+}^{\varphi}(q)^{2}-P_{+}^{\varphi}(k)^{2}}{2 a^{2} P_{+}^{\varphi}(k) P_{+}^{\varphi}(q)}\right) \delta^{(n-1)}\left(\vec{P}^{\varphi}(q)-\frac{P_{+}^{\varphi}(q)}{P_{+}^{\varphi}(k)} \vec{P}^{\varphi}(k)\right) \\
=(2 \pi)^{n} \frac{\delta\left(P_{+}^{\varphi}(k)-P_{+}^{\varphi}(q)\right)}{\left|-\frac{P_{+}^{\varphi}(k)^{2}+P_{+}^{\varphi}(q)^{2}}{2 a^{2} P_{+}^{\varphi}(q) P_{+}^{\varphi}(k)^{2}}(-a)\right|_{P_{+}^{\varphi}(k)=P_{+}^{\varphi}(q)}} \delta^{(n-1)}\left(\vec{P}^{\varphi}(q)-\frac{P_{+}^{\varphi}(q)}{P_{+}^{\varphi}(k)} \vec{P}^{\varphi}(k)\right)
\end{gathered}
$$




$$
=(2 \pi)^{n} a P_{+}^{\varphi}(q) \delta\left(P_{+}^{\varphi}(k)-P_{+}^{\varphi}(q)\right) \delta^{(n-1)}\left(\vec{P}^{\varphi}(q)-\vec{P}^{\varphi}(k)\right) .
$$

Here, in calculating the above expression, one zero of the function in the argument has been rejected due to the requirement that $P_{+}^{\varphi}<0$. This condition is obvious from the form of $P_{+}^{\varphi}$ in (87). Since the final goal is to express the result in terms of $P_{0}^{\varphi}$ and $\vec{P}^{\varphi}$, we perform the following computation

$$
\begin{gathered}
\delta\left(P_{0}^{\varphi}(q)-P_{0}^{\varphi}(k)\right)=\delta\left(\frac{1}{2} P_{+}^{\varphi}(q)-\frac{1}{2 a^{2} P_{+}^{\varphi}(q)}+\frac{\vec{P}^{\varphi}(q)^{2}}{2 P_{+}^{\varphi}(q)}-\frac{1}{2} P_{+}^{\varphi}(k)+\frac{1}{2 a^{2} P_{+}^{\varphi}(k)}-\frac{\vec{P}^{\varphi}(k)^{2}}{2 P_{+}^{\varphi}(k)}\right) \\
=\frac{\delta\left(P_{+}^{\varphi}(k)-P_{+}^{\varphi}(q)\right)}{\left|-\frac{1}{2}-\frac{1}{2 a^{2} P_{+}^{\varphi}(k)^{2}}+\frac{\vec{P}^{\varphi}(k)^{2}}{2 P_{+}^{\varphi}(k)^{2}}\right|_{P_{+}^{\varphi}(k)=P_{+}^{\varphi}(q)}}=\frac{\left|P_{+}^{\varphi}(q)\right|}{\left|\mathcal{P}_{n}^{\varphi}(q)\right|} \delta\left(P_{+}^{\varphi}(k)-P_{+}^{\varphi}(q)\right),
\end{gathered}
$$

where use has been made of the relation $\mathcal{P}_{n}^{\varphi}(k)=\frac{1}{2} P_{+}^{\varphi}(k)+\frac{1}{2 a^{2} P_{+}^{\varphi}(k)}-\frac{\vec{P}^{\varphi}(k)^{2}}{2 P_{+}^{\varphi}(k)}$. Inserting (91) in (90), we get

$$
(2 \pi)^{n} \delta^{(n)}\left(P^{\varphi}\left(k^{-1} \oplus q\right)\right)=(2 \pi)^{n} a\left|\mathcal{P}_{n}^{\varphi}(q)\right| \delta\left(P_{0}^{\varphi}(q)-P_{0}^{\varphi}(k)\right) \delta^{(n-1)}\left(\vec{P}^{\varphi}(q)-\vec{P}^{\varphi}(k)\right)
$$

and it can be recognized as

$$
(2 \pi)^{n} a\left|\mathcal{P}_{n}^{\varphi}(q)\right| \delta\left(P_{0}^{\varphi}(q)-P_{0}^{\varphi}(k)\right) \delta^{(n-1)}\left(\vec{P}^{\varphi}(q)-\vec{P}^{\varphi}(k)\right)=a \int d^{n} x\left(e^{i P^{\varphi}(k) \cdot x}\right)^{*}\left|\mathcal{P}_{n}^{\varphi}(q)\right| e^{i P^{\varphi}(q) \cdot x} .
$$

Finally, the result we have obtained is

$$
\int d^{n} x\left(e^{i P^{\varphi}(k) \cdot x}\right)^{\dagger} \star_{N} e^{i P^{\varphi}(q) \cdot x}=\int d^{n} x\left(e^{i P^{\varphi}(k) \cdot x}\right)^{*} \sqrt{1+a^{2} \partial_{\mu} \partial^{\mu}} e^{i P^{\varphi}(q) \cdot x} .
$$

In the last step, we have utilized the identity $\quad a\left|\mathcal{P}_{n}^{\varphi}\right|=\sqrt{1-a^{2} P^{\varphi}}=\sqrt{1+a^{2} D^{\mu} D_{\mu}}$ and the fact that we are working in the natural realization where $D_{\mu} \equiv \partial_{\mu}$. This proves the relation (84) which is a generalization of the integral identity found in [30]. Here it was proved by starting from the general ordering prescription characterized by the function $\varphi$, in contrast to Ref. 30] where the same relation is obtained by starting from some particular ordering prescription, namely, the right one.

We can now look upon the action for a free massive scalar field on noncommutative $\kappa$-deformed Minkowski space

$$
S=\int d^{4} x\left[\frac{1}{2}\left(\partial_{\mu} \phi\right)^{\dagger} \star_{N}\left(\partial^{\mu} \phi\right)(x)+\frac{m^{2}}{2} \phi^{\dagger} \star_{N} \phi(x)\right] .
$$

The construction of this action is based on the star product corresponding to the natural realization. At this place we point out that the action (25) is $\kappa$-Poincare invariant. Namely, in the natural realization, where $D_{\mu}=\partial_{\mu}$, the Dirac derivative $D_{\mu}$ is a vector under Lorentz algebra and the coproduct $\triangle_{N}\left(D_{\mu}\right)$ transforms in a covariant way [25]. This coproduct (for the Euclidean case) is given by

$$
\triangle_{N}\left(D_{\mu}\right)=D_{\mu} \otimes Z^{-1}+1 \otimes D_{\mu}+i a_{\mu} D_{\lambda} Z \otimes D_{\lambda}-\frac{i a_{\mu}}{2} \square Z \otimes\left(i a_{\lambda} D_{\lambda}\right),
$$

where

$$
Z^{-1}=-i a_{\lambda} D_{\lambda}+\sqrt{1+a^{2} D_{\lambda} D_{\lambda}}
$$

The last equation represents an invariant shift operator [25]. From expressions (see Eq.(64))

$$
\left(f \star_{N} g\right)(u)=\left.\mu\left(e^{u_{\alpha}\left(\triangle_{N}-\triangle_{0}\right) \partial_{\alpha}}(f \otimes g)(x)\right)\right|_{x=u} .
$$

and

$$
e^{i k \cdot x} \star_{N} e^{i q \cdot x}=e^{i \triangle_{N}(k, q) \cdot x}
$$


we conclude that $\star_{N}$ product in natural realization (where $D_{\mu}=\partial_{\mu}$ ) is noncommutative, nonlocal, but $\kappa$-Poincare invariant. Hence, the star product $\star_{N}$ of two scalar fields is scalar with respect to $\kappa$-Poincare symmetry. Similarly, from the same argument, the term $D_{\mu} \phi \star_{N} D^{\mu} \phi$ is also invariant under the action of $\kappa$-Poincare symmetry. As a consequence of these consideration, we conclude that the action (95) is invariant under the Poincare algebra (see also Eq.(84)) and $\kappa$-Poincare symmetry.

A few comments are in order regarding expression (95) . Considering the action (95) or the expression (94) obtained by spliting (95) to Fourier components, we see that the construction of the action can be basicaly taken along two different routes. The first one keeps the realizations of 4-momenta appearing in (94) fixed, while simultaneously changing the ordering prescription which the star product should be associated to. The second route is based on choosing the convenient ordering prescription with its accompanying star product and keeping it fixed, while simultaneously changing the realizations of 4-momenta appearing in the exponentials of (94) and used to define $\kappa$-Minkowski space. Here we adopt the second approach, where we choose the star product corresponding to natural realization and keep it fixed. This realization of the deformed space has special properties which enable us to cast the theory described by the action (95) in a form which is easier to handle and which is closer in form to theories we know how to deal with. With the help of the identity (84) relating the integration of the star product of fields to the integration of the standard pointwise product of fields, the action (95) can be cast in the form

$$
S=\int d^{4} x\left[\frac{1}{2}\left(\partial_{\mu} \phi\right)^{*} \sqrt{1+a^{2} \partial_{\mu} \partial^{\mu}}\left(\partial^{\mu} \phi\right)(x)+\frac{m^{2}}{2} \phi^{*} \sqrt{1+a^{2} \partial_{\mu} \partial^{\mu}} \phi(x)\right] .
$$

This shows that the free scalar field theory on $\kappa$-deformed Minkowski space is equivalent to a nonlocal, relativistically invariant free scalar field theory on Minkowski spacetime. Also, we see that once the ordering prescription together with its noncommutative star product is chosen and fixed, the resulting action (98) is the same, independent of the "gauge" function $\varphi$, regardless of the realization of 4-momenta in the corresponding NC space. Although the theory, through the square root, has higher derivatives, thus being nonlocal, it poses no significant physical effects as long as the interaction is not included. Particularly, we expect that there will be no new poles leading to the problems with unitarity. However, at the quantum level one would come up to the necessity for the change in statistics, causing the situation to be more complex.

It is worth mentioning that the $\kappa$-type of the space deformation can also be approached on the basis of an alternative general treatment [43] of twists with the star products. This includes the investigation of the particular type of the map, deforming the commutative subalgebra into a noncommutative one. The deformation map of this kind has already been applied [43] to yield the $n$-dimensional Moyal plane, as well as the Manin plane.

At the end, let us make some comments. In the present paper, we have started from the $\kappa$-deformed algebra (3) and considered its extension to the extended Lorentz algebra where we have included the generators of rotations and the covariant Dirac derivative $D_{\mu}$. The requirement that the Lorentz algebra is undeformed caused the deformed Heisenberg algebra (15) to be uniquelly determined. For this deformed Heisenberg algebra there exists a unique natural realization, specified by the requirement $D_{\mu} \equiv \partial_{\mu}$, and given by the relations (19), i.e. (70). There is also a unique ordering prescription corresponding to this realization. It was refered to as a natural ordering and it was specified by (75). We were also found the coproduct and the star product $\star_{N}$ corresponding to the natural realization. The star product $\star_{N}$ has been shown to obey the relation (78) which implied the following property of $\star_{N}$ to be satisfied

$$
\begin{gathered}
\int d^{n} x \phi_{\varphi}^{\dagger}(x) \star_{N} \psi_{\varphi}(x)=\int d^{n} k \tilde{\phi}^{*}(k) \int d^{n} q \tilde{\psi}(q) \int d^{n} x\left(e^{i P^{\varphi}(k) \cdot x}\right)^{\dagger} \star_{N} e^{i P^{\varphi}(q) \cdot x} \\
=\int d^{n} k \tilde{\phi}^{*}(k) \int d^{n} q \tilde{\psi}(q) \int d^{n} x: e^{i k^{-1} \cdot \hat{x}^{N a t}}::_{\varphi}: e^{i q \cdot \hat{x}^{N a t}}: \varphi|0\rangle=\int d^{n} x \phi_{\varphi}^{*}(x) \chi(\partial) \psi_{\varphi}(x),
\end{gathered}
$$

where, according to (84), the function $\chi$ is $\chi(\partial)=\sqrt{1+a^{2} \partial^{2}}$. In writing the Fourier expansions of the fields $\phi$ and $\psi$, a particular realization $\varphi$ of $\mathrm{NC}$ space was used, but the final result, i.e. the function $\chi$ does not depend 
on the realization $\varphi$. In the steps undertaken in (99) (which are, in fact, concise summary of the calculations done earlier in the paper, see particularly Eqs.(73), (83) and the consideration after that), we have utilised the following field expansion

$$
\phi_{\varphi}(x)=\int d^{n} x \tilde{\phi}(k) e^{i P^{\varphi}(k) \cdot x} .
$$

It is consistent, through the relation (73), with the Fourier expansion (22)

$$
\phi_{\varphi}(\hat{x})=\int d^{n} x \tilde{\phi}(k): e^{i k \cdot \hat{x}}:_{\varphi}
$$

and the relation $\phi_{\varphi}\left(\hat{x}^{N a t}\right)|0\rangle=\phi_{\varphi}^{N a t}(x) \equiv \phi_{\varphi}(x)$.

It is important to emphasize that we could equally well start our consideration from an arbitrary deformed Heisenberg algebra

$$
\left[D_{\mu}, \hat{x}_{\nu}\right]=\varphi_{\mu \nu}(D)
$$

where $\varphi_{\mu \nu}$ is some function compatible with the commutation relations (1), and take the same steps as those performed for the algebra (15). We would also find the unique natural realization (characterized by $D_{\mu} \equiv \partial_{\mu}$ and the mapping $\hat{x}_{\mu}^{N a t}=x_{\alpha} \varphi_{\alpha \mu}(\partial)$ which has to satisfy the commutation relations (1)) and natural ordering corresponding to the algebra (100). There would also be a coproduct and a star product $\star_{N}$ which could be associated with (100). One would find that the specific property of the star product $\star_{N}$, corresponding to (100), is also enclosed in the identity (99), except for the fact that the function $\chi$ now has a form appropriate to the case in consideration.

The adventage of our approach is in a simple construction of $\kappa$-Poincare action using the notion of natural realization. This route of consideration has an advantage that it provides a direct way for constructing any $\kappa$ Poincare invariant action. The Dirac derivative $D_{\mu}$, Eq.(13), transforms as a vector and the right hand sides of the commutation relations (9), (10) and (15) have covariant form. Hence, the coproducts $\triangle\left(D_{\mu}\right)$ and $\triangle\left(M_{\mu \nu}\right)$ have also covariant forms under $\kappa$-Poincare symmetry. Thus we have that starting with generators of undeformed Poincare algebra, the right hand sides of (9),(10) and (15) and the corresponding coproducts $\triangle\left(D_{\mu}\right)$ and $\triangle\left(M_{\mu \nu}\right)$ are covariant under the twisted Poincare symmetry.

In papers [20], 26], 27] the symmetric realization corresponding to symmetric Weyl ordering was used, but therein $\triangle\left(D_{\mu}\right)$ and $\triangle\left(M_{\mu \nu}\right)$ are given by Eqs.(11), (13) with $\varphi(A)=\frac{A}{e^{A}-1}$, whereas in our approach, which uses the notion of natural realization, $D_{\mu}$ and $M_{\mu \nu}$ have particularly simple form [25], namely $D_{\mu}=\partial_{\mu}$ and $M_{\mu \nu}=x_{\mu} \partial_{\nu}-x_{\nu} \partial_{\mu}$. Hence, both realizations are covariant and equivalent. In analogy with the gauge theory they correspond to different covariant gauge conditions. Finaly, in contrast to [20], 27], where the deformed Klein-Gordon operator has the form $\square+m^{2}=\frac{2}{a^{2}}\left(1-\sqrt{1-a^{2} D^{2}}\right)+m^{2}$, we take the simplest possibility for the Klein-Gordon operator by choosing

$\square+m^{2}=D^{2}+m^{2}$. The physical consequences of this approach are still under investigation and will be reported in near future.

\section{CONCLUSION}

We have constructed $\kappa$-deformed noncommutative space-time of the Lie algebra type with undeformed extended Lorentz algebra and deformed coalgebra by realizing noncommutative coordinates, generalized derivatives and generalized rotation generators in terms of the commutative coordinates and their derivatives. The coproducts of the generalized rotation generators and of the Dirac derivatives, respectively, have been identified. A unique correspondence between the particular realization of $\kappa$-deformed space-time and the corresponding ordering prescription has been found. For fixed deformed Heisenberg algebra (15) all these realizations of $\kappa$-deformed Minkowski space are of equal importance, none of the realizations is preferable to the others, implying that physical results do not depend on the particular realization chosen. Accordingly, the choice of the ordering prescription or the choice of a particular realization is analogous to the situation in gauge theory where a particular gauge is chosen. We have found the 
expression for the star product on $\kappa$-Minkowski space corresponding to an arbitrary "gauge" function $\varphi$ defining the realization. This result is related to the important relation between a coproduct and a star product in terms of an exponential map.

Although all realizations are on equal footing, for constructing a field theory on $\kappa$-deformed Minkowski space, we have used a particular realization, the natural realization, which is not contained within the set of realizations parametrized by the "gauge" function $\varphi$, but which was shown to have some preferable properties that have enabled us to reduce the original field theory on $\kappa$-deformed space to the nonlocal, relativistically invariant, scalar field theory on Minkowski space-time. The result of this reduction has also been shown to not depend on the realization/ordering taken for $\kappa$-Minkowski space. Thus to conclude, there is one particular noncommutative star product on the algebra of functions, namely the one that corresponds to the natural realization. Once this star product is fixed, the realization of $\mathrm{NC}$ space alone is not important, i.e. changing the realizations of 4-momenta and coordinates used to define the $\kappa$-Minkowski space does not influence the final result for the action. The line of consideration which is carried out here may appear useful in $2+1$ dimensional models of quantum gravity where the corresponding Lie algebra is $S U(2)$ or $S U(1,1)$, 44]. Another possible route for future investigations is to consider other noncommutative space-times. We believe that the line of analysis presented here is applicable to other noncommutative spacetimes, particularly those of Lie-algebra type.

\section{Acknowledgment}

We thank T.R.Govindarajan for reading the manuscript and for helpful comments. This work was supported by the Ministry of Science and Technology of the Republic of Croatia under contract No. 098-0000000-2865. This work was done within the framework of the Indo-Croatian Joint Programme of Cooperation in Science and Technology sponsored by the Department of Science and Technology, India, and the Ministry of Science, Education and Sports, Republic of Croatia.

\section{Appendix A. DERIVATION OF SOME USEFUL IDENTITIES I}

Here, we shall derive some usefull identities that are used in calculations taking place in sections 2 and 3 , where the T-operator and the star product corresponding to $\varphi$-realization of $\kappa$-noncommutative space were found. First we prove the following identity:

$$
: N^{k}:: N^{l}:=\sum_{r=0}^{\min (k, l)} r !\left(\begin{array}{l}
k \\
r
\end{array}\right)\left(\begin{array}{l}
l \\
r
\end{array}\right): N^{k+l-r}:
$$

where $N \equiv \sum_{i=1}^{N-1} x_{i} \partial_{i}$ is the number operator and the symbol :: has the usual meaning of normal ordering with all $x_{i}$ coming to the left with respect to all $\partial_{i}$. The variables $x_{i}$ and $\partial_{i}$ satisfy the ordinary Heisenberg algebra

$$
\left[\partial_{i}, x_{j}\right]=\delta_{i j}, \quad i, j=1, \ldots, N-1 .
$$

The proof of A. 1) will be done by induction. For the base of induction we have $: N:: N:=x_{i} \partial_{i}+x_{i} x_{j} \partial_{i} \partial_{j}=: N:$ $+: N^{2}$ : and this is exactly what we get from (A. 1) by taking $l=k=1$. For the step of induction, we assume that (A. 1)holds for some generic $l, k$ and consider the expression $: N^{k}:: N^{l+1}:$. It can be transformed by using the identity

$$
: N^{k}: x_{i}=x_{i}: N^{k}:+k x_{i}: N^{k-1}:
$$

and with the help of this identity we proceed as follows:

$$
: N^{k}:: N^{l+1}:=\sum_{i=1}^{N-1}\left(x_{i}: N^{k}:+k x_{i}: N^{k-1}:\right): N^{k}: \partial_{i}
$$




$$
\begin{gathered}
=\sum_{i=1}^{N-1} x_{i} \sum_{r=0}^{\min (k, l)} r !\left(\begin{array}{l}
k \\
r
\end{array}\right)\left(\begin{array}{l}
l \\
r
\end{array}\right): N^{k+l-r}: \partial_{i}+k \sum_{i=1}^{N-1} x_{i} \sum_{r=0}^{\min (k-1, l)} r !\left(\begin{array}{c}
k-1 \\
r
\end{array}\right)\left(\begin{array}{l}
l \\
r
\end{array}\right): N^{k+l-r-1}: \partial_{i} \\
=\sum_{r=0}^{\min (k, l)} r !\left(\begin{array}{l}
k \\
r
\end{array}\right)\left(\begin{array}{l}
l \\
r
\end{array}\right): N^{k+l+l-r}:+k \sum_{r=0}^{\min (k-1, l)} r !\left(\begin{array}{c}
k-1 \\
r
\end{array}\right)\left(\begin{array}{l}
l \\
r
\end{array}\right): N^{k+l-r}: .
\end{gathered}
$$

If we take $k<l$ and change the summation index, after rearranging the sums, we get

$$
\begin{gathered}
\sum_{r=0}^{k} r !\left(\begin{array}{l}
k \\
r
\end{array}\right)\left(\begin{array}{l}
l \\
r
\end{array}\right): N^{k+l+l-r}:+\sum_{r=1}^{k} k(r-1) !\left(\begin{array}{l}
k-1 \\
r-1
\end{array}\right)\left(\begin{array}{c}
l \\
r-1
\end{array}\right): N^{k+l+1-r}: \\
=: N^{k+l+l}:+\sum_{r=1}^{k} r !\left(\begin{array}{l}
k \\
r
\end{array}\right)\left[\left(\begin{array}{l}
l \\
r
\end{array}\right)+\left(\begin{array}{c}
l \\
r-1
\end{array}\right)\right]: N^{k+l+1-r}:=\sum_{r=0}^{\min (k, l+1)} r !\left(\begin{array}{c}
k \\
r
\end{array}\right)\left(\begin{array}{c}
l+1 \\
r
\end{array}\right): N^{k+l+1-r}:,
\end{gathered}
$$

which is the identity (A. 1) for $l \rightarrow l+1$. The analogous procedure can be carried out for $l<k$ and also for the expression : $N^{k+1}:: N^{l}$ : leading to the same conclusion, which completes the proof.

With the help of the identity (A. 1) we can now prove the following important relation:

$$
: e^{N A}:: e^{N B}:=: e^{N(A+B+A B)}:
$$

which is the identity for the normally ordered exponentials and $A, B$ are arbitrary operators commuting with the number operator $N$. We proceed as follows:

$$
\begin{gathered}
: e^{N A}:: e^{N B}:=\sum_{l=0}^{\infty} \sum_{k=0}^{\infty} \frac{1}{l ! k !}: N^{l}:: N^{k}: A^{l} B^{k}=\sum_{l=0}^{\infty} \sum_{k=0}^{\infty} \frac{1}{l ! k !} \sum_{r=0}^{\min (k, l)} r !\left(\begin{array}{l}
l \\
r
\end{array}\right)\left(\begin{array}{l}
k \\
r
\end{array}\right): N^{l+k-r}: A^{l} B^{k} \\
=\sum_{s=0}^{\infty} \sum_{k=0}^{s} \sum_{r=0}^{\min (k, s-k)} \frac{1}{(s-k-r) !} \frac{1}{r !(k-r) !}: N^{s-r}: A^{s-k} B^{k},
\end{gathered}
$$

where in the last line we have skipped to the new summation variable $s=k+l$. To accomodate for the upper bound of the 3rd sum in the last line of (A. 6), we split the above expression into two parts

$$
\begin{gathered}
: e^{N A}:: e^{N B}:=\sum_{s=0}^{\infty}\left(\sum_{k=0}^{\left[\frac{s}{2}\right]} \sum_{r=0}^{k} \frac{1}{(s-k-r) !} \frac{1}{r !(k-r) !}: N^{s-r}: A^{s-k} B^{k}+\sum_{k=\left[\frac{s}{2}\right]+1}^{s} \sum_{r=0}^{s-k} \frac{1}{(s-k-r) !} \frac{1}{r !(k-r) !}: N^{s-r}: A^{s-k} B^{k}\right) \\
=\sum_{s=0}^{\infty}\left(\sum_{r=0}^{\left[\frac{s}{2}\right]} \sum_{k=r}^{\left[\frac{s}{2}\right]} \frac{1}{(s-k-r) !} \frac{1}{r !(k-r) !}: N^{s-r}: A^{s-k} B^{k}+\sum_{r=0}^{s-\left[\frac{s}{2}\right]-1} \sum_{k=\left[\frac{s}{2}\right]+1}^{s-r} \frac{1}{(s-k-r) !} \frac{1}{r !(k-r) !}: N^{s-r}: A^{s-k} B^{k}\right) \\
=\sum_{s=0}^{\infty} \sum_{r=0}^{\left[\frac{s}{2}\right]} \sum_{k=r}^{s-r} \frac{1}{(s-k-r) !} \frac{1}{r !(k-r) !}: N^{s-r}: A^{s-k} B^{k},
\end{gathered}
$$

with the symbol [] denoting the lowest integer. Under the agreement that the terms with negative integers in factorials drop out, the region of summation in (A. 7) can be extended to include $s$ as the upper bound in the last two sums of (A. 7). The expression A. 7) now becomes

$$
\sum_{s=0}^{\infty} \sum_{r=0}^{s} \sum_{k=r}^{s} \frac{1}{(s-k-r) !} \frac{1}{r !(k-r) !}: N^{s-r}: A^{s-k} B^{k}=\sum_{m=0}^{\infty} \frac{: N^{m}:}{m !} \sum_{r=0}^{\infty} \sum_{k=r}^{m+r} \frac{1}{(m-k) !} \frac{m !}{r !(k-r) !} A^{m+r-k} B^{k}
$$




$$
=\sum_{m=0}^{\infty} \frac{N^{m}}{m !} \sum_{r=0}^{\infty} \sum_{a=0}^{m} \frac{1}{(m-r-a) !} \frac{m !}{r ! a !} A^{m-r-a} B^{a}(A B)^{r} .
$$

Here, in the first step we have made the change of the summation variable as $s=r+m$ and then, in the second step (the second line of (A. 8) ) we skip to the summation variable $a=k-r$. Note that in the expressions (A. 8) it is understood that the terms with factorials whose arguments are negative drop out. Making use of this fact, the upper bound of the $r$-summation in (A. 8) can be reduced to $m$, (for $r>m$, the corresponding terms drop out anyway) which finally gives

$$
\begin{gathered}
: e^{N A}:: e^{N B}:=\sum_{m=0}^{\infty} \frac{: N^{m}:}{m !} \sum_{r=0}^{m} \sum_{a=0}^{m} \frac{m !}{(m-r-a) ! r ! a !} A^{m-r-a} B^{a}(A B)^{r} \\
=\sum_{m=0}^{\infty} \frac{: N^{m}:}{m !}(A+B+A B)^{m}=: e^{N(A+B+A B)}:
\end{gathered}
$$

where, in the transition between the first and the second line, we have utilized the multinomial expansion

$$
(a+b+c)^{n}=\sum_{n_{1}=0}^{n} \sum_{\substack{n_{2}=0 \\ n_{1}+n_{2}+n_{3}=n}}^{n} \sum_{n_{3}=0}^{n} \frac{n !}{n_{1} ! n_{2} ! n_{3} !} a^{n_{1}} b^{n_{2}} c^{n_{3}} .
$$

Finally, it remains to prove the relation

$$
N^{k}=\sum_{l=0}^{k} \sum_{p=0}^{l} \frac{(-1)^{l-p} p^{k}}{p !(l-p) !}: N^{l}: \equiv \sum_{l=0}^{k} c_{l, k}: N^{l}:
$$

It can be again done by induction. From (A.11) we see that the coefficients $c_{l, k}$ are defined as

$$
c_{l, k}=\sum_{p=0}^{l} \frac{(-1)^{l-p} p^{k}}{p !(l-p) !} .
$$

While the base of the induction is trivial, for the step of induction we have

$$
N^{k+1}=N \cdot N^{k}=\sum_{l=0}^{k} c_{l, k}: N:: N^{l}:=\sum_{l=0}^{k} c_{l, k}\left[: N^{l+1}:+l: N^{l}:\right],
$$

where use has been made of the identity (A. 1). In order to complete the step of induction, the expression (A. 13) should be equal to $\sum_{l=0}^{k+1} c_{l, k+1}: N^{l}$,, which implies that the following recursive relations have to be satisfied:

$$
\begin{gathered}
c_{k+1, k+1}=c_{k, k}=c_{0,0}=1, \quad c_{0, k}=0 \quad \text { for } \quad k \neq 0, \\
c_{l, k+1}=c_{l-1, k}+l c_{l, k}, \quad k>0 .
\end{gathered}
$$

The coefficients (A. 12) are easily shown to satisfy the recursions (A. 14) and this completes the proof of the relation (A. 11).

Now, (A. 11) can be inverted to obtain

$$
: N^{k}:=k !\left(\begin{array}{c}
N \\
k
\end{array}\right)
$$

Using the above identity, we have for an arbitrary operator $f$, commuting with the number operator $N$

$$
: e^{N f}:=\sum_{k=0}^{\infty} \frac{1}{k !}: N^{k}: f^{k}=\sum_{k=0}^{\infty}\left(\begin{array}{c}
N \\
k
\end{array}\right) f^{k}=(1+f)^{N}=e^{N \ln (1+f)}=e^{N F},
$$

where $f=e^{F}-1$. That is, we have

$$
: e^{N\left(e^{F}-1\right)}:=e^{N F}
$$




\section{Appendix B. DERIVATION OF SOME USEFUL IDENTITIES II}

In this section we prove that the relation

$$
\varphi\left(A_{w}\right) \lim _{\substack{u \rightarrow w \\ t \rightarrow w}} f(u) g(t)=\lim _{\substack{u \rightarrow w \\ t \rightarrow w}} \varphi\left(A_{u}+A_{t}\right) f(u) g(t)
$$

holds. We begin with

$$
\begin{gathered}
\varphi\left(A_{w}\right) \lim _{\substack{u \rightarrow w \\
t \rightarrow w}} f(u) g(t)=\sum_{k=0}^{\infty} \frac{1}{k !}\left(\frac{\partial^{k} \varphi}{\partial A_{w}^{k}}\right)_{A_{w}=0}(i a)^{k} \frac{\partial^{k}}{\partial w_{n}^{k}}(f(w) g(w)) \\
=\sum_{k=0}^{\infty} \frac{1}{k !}\left(\frac{\partial^{k}}{\partial A_{w}^{k}}\right)_{A_{w}=0}(i a)^{k} \sum_{m=0}^{k}\left(\begin{array}{c}
k \\
m
\end{array}\right) \frac{\partial^{m} f(w)}{\partial w_{n}^{m}} \frac{\partial^{k-m} g(w)}{\partial w_{n}^{k-m}}=\sum_{k=0}^{\infty} \sum_{m=0}^{k} \frac{(i a)^{k}}{k !}\left(\frac{\partial^{k} \varphi}{\partial A_{w}^{k}}\right)_{A_{w}=0}\left(\begin{array}{c}
k \\
m
\end{array}\right) \frac{\partial^{m} f(w)}{\partial w_{n}^{m}} \frac{\partial^{k-m} g(w)}{\partial w_{n}^{k-m}} .
\end{gathered}
$$

From the other side, we have

$$
\begin{gathered}
\lim _{\substack{u \rightarrow w \\
t \rightarrow w}} \varphi\left(A_{u}+A_{t}\right) f(u) g(t)=\lim _{\substack{u \rightarrow w \\
t \rightarrow w}} \sum_{k=0}^{\infty} \frac{1}{k !}\left(\frac{\partial^{k} \varphi}{\partial\left(A_{u}+A_{t}\right)^{k}}\right)_{A_{u}+A_{t}=0}\left(A_{u}+A_{t}\right)^{k} f(u) g(t) \\
=\lim _{\substack{u \rightarrow w \\
t \rightarrow w}} \sum_{k=0}^{\infty} \frac{1}{k !}\left(\frac{\partial^{k} \varphi}{\partial\left(A_{u}+A_{t}\right)^{k}}\right)_{A_{u}+A_{t}=0} \sum_{m=0}^{k}\left(\begin{array}{c}
k \\
m
\end{array}\right) A_{u}^{m} A_{t}^{k-m} f(u) g(t) \\
=\sum_{k=0}^{\infty} \sum_{m=0}^{k} \frac{(i a)^{k}}{k !}\left(\frac{\partial^{k} \varphi}{\partial\left(2 A_{w}\right)^{k}}\right)_{2 A_{w}=0}\left(\begin{array}{c}
k \\
m
\end{array}\right) \frac{\partial^{m} f(w)}{\partial w_{n}^{m}} \frac{\partial^{k-m} g(w)}{\partial w_{n}^{k-m}}
\end{gathered}
$$

and this is identical with (B. 2), which completes the proof.

[1] S. Doplicher, K. Fredenhagen and J. E. Roberts, The quantum structure of spacetime at the Planck scale and quantum fields, Phys. Lett. B331, 39 (1994); Commun. Math. Phys. 172, 187 (1995) hep-th/0303037.

[2] N. Seiberg and E. Witten, String theory and noncommutative geometry, JHEP 09, 032 (1999) hep-th/9908142]; J. de Boer, P. A. Grassi and P. van Nieuwenhuizen, Noncommutative superspace from string theory, Phys. Lett. B574, 98 (2003) hep-th/0302078.

[3] M. R. Douglas and N. A. Nekrasov, Noncommutative field theory, Rev. Mod. Phys. 73, 977 (2001) hep-th/0106048.

[4] A.P. Balachandran, T.R. Govindarajan, C. Molina and P. Teotonio-Sobrinho, JHEP 0410, 72 (2004); A.P. Balachandran, T.R. Govindarajan, A.G. Martins and P. Teotonio-Sobrinho, JHEP 0411, 68 (2004).

[5] R. J. Szabo, Quantum field theory on noncommutative spaces, Phys. Rept. 378, 207 (2003) hep-th/0109162]; R. J. Szabo, "Symmetry, gravity and noncommutativity, Class. Quant. Grav. 23 (2006) R199 arXiv:hep-th/0606233.

[6] P. Aschieri, B. Jurco, P. Schupp and J. Wess, Noncommutative GUTs, standard model and C,P,T ; Nucl. Phys. B651 45 (2003) hep-th/0205214; P. Aschieri, C. Blohmann, M. Dimitrijević, F. Meyer, P. Schupp and J. Wess, A gravity theory on noncommutative spaces, Class. Quant. Grav. 223511 (2005) hep-th/0504183; A.P. Balachandran, T.R. Govindarajan, Kumar S. Gupta and Seckin Kurkcuoglu, Class. Quant. Grav. 23, 5799 (2006); X. Calmet and A. Kobakhidze, "Noncommutative general relativity," Phys. Rev. D 72 (2005) 045010 arXiv:hep-th/0506157.

[7] J. Lukierski, A. Nowicki, H. Ruegg and V. N. Tolstoy, Q-deformation of Poincaré algebra, Phys. Lett. B264, 331 (1991).

[8] J. Lukierski, A. Nowicki and H. Ruegg, New quantum Poincaré algebra, and $\kappa$-deformed field theory, Phys. Lett. B293, 344 (1992).

[9] J. Lukierski and H. Ruegg, Quantum $\kappa$-Poincaré in any dimension, Phys. Lett. B329, 189 (1994) hep-th/9310117.

[10] S. Majid and H. Ruegg, Bicrossproduct structure of $\kappa$-Poincaré group and noncommutative geometry, Phys. Lett. B334, 348 (1994) hep-th/9404107.

[11] P. Kosiński and P. Maślanka, The duality between $\kappa$-Poincaré algebra and $\kappa$-Poincaré group, [ hep-th/9411033.

[12] A. Sitarz, "Noncommutative differential calculus on the kappa Minkowski space," Phys. Lett. B 349 (1995) 42 arXiv:hep-th/9409014. 
[13] K. Kosiński, J. Lukierski and P. Maślanka, Local $D=4$ field theory on $\kappa$-Minkowski space, Phys. Rev. D62, 025004 (2000) hep-th/9902037.

[14] K. Kosiński, J. Lukierski and P. Maślanka, Local field theory on $\kappa$-Minkowski space, «-products and noncommutative translations, Czech. J. Phys. 50, 1283 (2000) [ hep-th/0009120].

[15] P. Kosiński, J. Lukierski, P. Maślanka and A. Sitarz, Generalised $\kappa$-deformations and deformed relativistic scalar fields on noncommutative Minkovski space, [hep-th/0307038].

[16] G. Amelino-Camelia, "Testable scenario for relativity with minimum-length," Phys. Lett. B 510 (2001) 255 arXiv:hep-th/0012238; G. Amelino-Camelia, "Relativity in space-times with short-distance structure governed by an observer-independent (Planckian) length scale," Int. J. Mod. Phys. D 11 (2002) 35 arXiv:gr-qc/0012051]; N. R. Bruno, G. Amelino-Camelia and J. Kowalski-Glikman, "Deformed boost transformations that saturate at the Planck scale," Phys. Lett. B 522 (2001) 133 arXiv:hep-th/0107039.

[17] J. Kowalski-Glikman and S. Nowak, "Doubly special relativity theories as different bases of kappa-Poincare algebra," Phys. Lett. B 539 (2002) 126 arXiv:hep-th/0203040.

[18] G. Amelino-Camelia and M. Arzano, Coproduct and star-product in field theories on Lie algebra noncomutative spacetime, Phys. Rev. D65, 084044 (2002) hep-th/0105120.

[19] G. Amelino-Camelia, F. D'Andrea and G. Mandanici, Group velocity in noncommutative spacetime, JCAP 0309 (2003) 006 hep-th/0211022.

[20] M. Dimitrijević, L. Jonke, L. Möller, E. Tsouchnika, J. Wess and M. Wohlgenannt, Deformed field theory on $\kappa$-spacetime, Eur. Phys. J. C31 129 (2003) hep-th/0307149.

[21] M. Dimitrijević, F. Meyer, L. Möller and J. Wess, Gauge theories on the $\kappa$-Minkovski space time, Eur. Phys. J. C36, 117 (2004) hep-th/0310116.

[22] M. Dimitrijević, L. Möller and E. Tsouchnika, Derivatives, forms and vector fields on the $\kappa$-deformed Euclidean space, J. Phys. A 37, 9749 (2004) hep-th/0404224.

[23] C. Jambor and A. Sykora, "Realization of algebras with the help of *-products," arXiv:hep-th/0405268;A. Sykora, "The application of *-products to noncommutative geometry and gauge theory," arXiv:hep-th/0412012.

[24] S. Meljanac and M. Stojic, "New realizations of Lie algebra kappa-deformed Euclidean space," Eur. Phys. J. C 47 (2006) 531 arXiv:hep-th/0605133.

[25] S. Meljanac, S. Kresic-Juric and M. Stojic, "Covariant realizations of kappa-deformed space," arXiv:hep-th/0702215, to appear in EPJC.

[26] L. Moller, "A symmetry invariant integral on kappa-deformed spacetime," JHEP 0512 (2005) 029 arXiv:hep-th/0409128.

[27] H. Grosse and M. Wohlgenannt, "On kappa-deformation and UV/IR mixing," Nucl. Phys. B 748 (2006) 473 arXiv:hep-th/0507030.

[28] A. Agostini, G. Amelino-Camelia, M. Arzano and F. D'Andrea, "Action functional for kappa-Minkowski noncommutative spacetime," arXiv:hep-th/0407227.

[29] A. Agostini, G. Amelino-Camelia, M. Arzano, A. Marciano and R. A. Tacchi, "Generalizing the Noether theorem for Hopfalgebra spacetime symmetries," arXiv:hep-th/0607221. M. Arzano and A. Marciano, "Symplectic geometry and Noether charges for Hopf algebra space-time symmetries," Phys. Rev. D 75 (2007) 081701 arXiv:hep-th/0701268; A. Agostini, F. Lizzi and A. Zampini, "Generalized Weyl systems and kappa-Minkowski space," Mod. Phys. Lett. A 17 (2002) 2105 arXiv:hep-th/0209174.

[30] L. Freidel, J. Kowalski-Glikman and S. Nowak, "From noncommutative kappa-Minkowski to Minkowski space-time," arXiv:hep-th/0612170.

[31] S. Ghosh, "The AdS particle," Phys. Lett. B 623 (2005) 251 arXiv:hep-th/0506084; S. Ghosh and P. Pal, "kappaMinkowski spacetime through exotic 'oscillator'," Phys. Lett. B 618 (2005) 243 arXiv:hep-th/0502192].

[32] S. Ghosh, "A Lagrangian for DSR particle and the role of noncommutativity," Phys. Rev. D 74 (2006) 084019 arXiv:hep-th/0608206]; S. Ghosh and P. Pal, "Deformed Special Relativity and Deformed Symmetries in a Canonical Framework," arXiv:hep-th/0702159.

[33] N. Durov, S. Meljanac, A. Samsarov and Z. Škoda, A universal formula for representing Lie algebra generators as formal power series with coefficients in the Weyl algebra, J.Algebra 309 (2007) 318 math.RT/0604096.

[34] D. Bonatsos and C. Daskaloyannis, General deformation schemes and $N=2$ supersymmetric quantum mechanics, Phys. Lett. B 307, 100 (1993); S. Meljanac, M. Mileković and S. Pallua, Unified view of deformed single-mode oscillator algebras, Phys. Lett. B328, 55 (1994) hep-th/9404039.

[35] S. Meljanac and M. Mileković, Unified view of multimode algebras with Fock-like representation, Int. J. Mod. Phys. A11, 1391 (1996).

[36] S. Meljanac and A. Perica, Number operators in a general quon algebra, J. Phys. A: Math. Gen. 27, 4737 (1994); S. Meljanac and A. Perica, Generalized quon statistics, Mod. Phys. Lett. A 9, 3293 (1994) hep-th/9409180; S. Meljanac, A. Perica and D. Svrtan, The energy operator for a model with a multiparametric infinite statistics, J. Phys. A 36, 6337 (2003) math-ph/0304038.

[37] V. Bardek and S. Meljanac, Deformed Heisenberg algebras, a Fock space representation and the Calogero model, Eur. Phys. J. C17 539 (2000) hep-th/0009099. V. Bardek, L. Jonke, S. Meljanac and M. Mileković, Calogero model, deformed oscillators and the collapse, Phys. Lett. B531 311 (2002) hep-th/0107053.

[38] S. Meljanac, M. Mileković and M. Stojić, Permutation invariant algebras, a Fock space realization and the Calogero model, Eur. Phys. J. C24 331 (2002) math-ph/0201061.

[39] L. Jonke and S. Meljanac, Bosonic realization of algebras in the Calogero model, Phys. Lett. B 526, 149 (2002) hep-th/0106135. 
[40] V. P. Nair and A. P. Polychronakos, Quantum mechanics on the noncommutative plane and sphere, Phys. Lett. B 505, 267 (2001) hep-th/0011172; L. Jonke and S. Meljanac, Representations of noncommutative quantum mechanics and symmerties, Eur. Phys. J. C 29, 433 (2003) hep-th/0210042; I. Dadić, L. Jonke and S. Meljanac, Harmonic oscillator on noncommutative spaces, Acta Phys. Slovaca 55, 149 (2005) hep-th/0301066.

[41] J. Wess, Deformed coordinates spaces; Derivatives, Lecture given at the Balkan workshop BW2003, August 2003, Vrnjačka Banja, Serbia, hep-th/0408080.

[42] M. Chaichian, P. P. Kulish, K. Nishijima and A. Tureanu, On a Lorentz-invariant interpretation on noncommutative space-time and its implications on noncommutative QFT, Phys. Lett. B604 98 (2004) hep-th/0408069. M. Chaichian, P. Presnajder and A. Tureanu, New concept of relativistic invariance in NC space-time: Twisted Poincaré symmetry and its implications, Phys. Rev. Lett. 94151602 (2005) hep-th/0409096.

[43] T.R.Govindarajan, private communication.

[44] L. Freidel and E. R. Livine, Ponzano-Regge model revisited III: Feynman diagrams and effective field theory, Class. Quant. Grav. 23, 2021 (2006) hep-th/0502106; L. Freidel and E. R. Livine, 3d quantum gravity and effective noncommutative quantum field theory, Phys. Rev Lett. 96, 221301 (2006) hep-th/0512113.

[45] L. Freidel and S. Majid, Noncommutative harmonic analysis, sampling theory and the Duflo map in $2+1$ quantum gravity, [hep/th-0601004]. 